\title{
EXTINCTION AND ERGODIC STATIONARY DISTRIBUTION OF A MARKOVIAN-SWITCHING PREY-PREDATOR MODEL WITH ADDITIONAL FOOD FOR PREDATOR
}

\author{
Xiaoxia Guo ${ }^{1,2}$ and Dehan Ruan ${ }^{3, *}$
}

\begin{abstract}
In this work we have studied a stochastic predator-prey model where the prey grows logistically in the absence of predator. All parameters but carrying capacity have been perturbed with telephone noise. The prey's growth rate and the predator's death rate have also been perturbed with white noises. Both of these noises have been proved extremely useful to model rapidly fluctuating phenomena Dimentberg (1988). The conditions under which extinction of predator and prey populations occur have been established. We also give sufficient conditions for positive recurrence and the existence of an ergodic stationary distribution of the positive solution, red which in stochastic predator-prey systems means that the predator and prey populations can be persistent, that is to say, the predator and prey populations can be sustain a quantity that is neither too much nor too little. In our analysis, it is found that the environmental noise plays an important role in extinction as well as coexistence of prey and predator populations. It is shown in numerical simulation that larger white noise intensity will lead to the extinction of the population, while telephone noise may delay or reduce the risk of species extinction.
\end{abstract}

Mathematics Subject Classification. 60G28, 34A12, 34K34.

Received April 26, 2019. Accepted November 20, 2019.

\section{INTRODUCTION}

Mathematical modelling in ecology has received great attention since the pioneer work of Lotka [20] and Volterra [27]. The importance of studying predator-prey model is to gain more insight into the complex processes and solve ecological problems, e.g., the control of populations of economically damaging species, particularly of agricultural weed and insect pests [9]. It is well documented that biological processes like disease [5], Allee effects [2, 19], migration [6] and alternative food sources [11], etc., have the potential to dramatically alter system dynamics. The role of predation is of major importance to ecologists as the ranges of large carnivores continue to collapse around the world. The dynamic predation behavior of a predator in an ecological system depends on functional response. The most common functional response in a prey-predator system is famous Holling type-II response being written as $f(N)=\frac{c N}{a+N}$, where $N$ is total number of prey individuals. The term $c$ represents the attack rate of predator on prey and parameter $a$ represents the half saturation constant [15].

Keywords and phrases: Markovian switching, prey-predator model, additional food, extinction, unique stationary distribution.

1 School of Mathematics and Information Sciences, Guangzhou University, Guangzhou 510006, PR China.

2 Department of Mathematics and Statistics, Concordia University, Montreal-H3G2W1, Canada.

3 Guangzhou International Institute of Finance and Guangzhou University, Guangzhou, Guangdong 510405, PR China.

* Corresponding author: mathhope@sina.com 
Most predator may not only eat a kind of food, for instance, wheat aphids and scale insect are the foods of coccinellid. Under resource limitation, predators may deviate from their usual diet and exhibit some inclination towards the alternative prey to prolong their survivability. In this context, many of experimental work and literatures addressing biological control consider prey as pest population and aims to control and eradicate the pest population through predation by providing additional food to the predator [25]. For example, Macrolophus pygmaeus is found to be an efficient natural enemy for the control of white flies and other small arthropod pests in Europe [23]. If $h_{N}$ represents handling time for each interaction between a predator and a prey, $e_{N}$ is a constant that depends on factors such as the predator's movement rate while searching or it's ability to detect the prey, the constant $h_{A}$ is the handling time of additional food biomass $A$ and $e_{A}$ represents the ability of the predator to capture the additional food, then the functional response with additional food may be written in the form $f(N)=\frac{c e_{N} N}{a+h_{A} e_{A} A+h_{N} e_{N} N}$, i.e., $f(N)=\frac{c_{1} N}{a_{1}+\alpha \eta A+N}$, where $\alpha=\frac{h_{A}}{h_{N}}, \eta=\frac{e_{A}}{e_{N}}, a_{1}=\frac{a}{h_{N} e_{N}}, c_{1}=\frac{c}{h_{N}}$. $\alpha=\frac{h_{A}}{h_{N}}$ signifies the quality of the additional food relative to the prey. If $h_{A}<h_{N}$ holds, then the predator can easily capture additional food than prey species and it implies that the additional food is of high quality. Therefore, $\alpha$ is less than 1 for high quality of additional food. $\eta=\frac{e_{A}}{e_{N}}$ represents effectual ability of the predator to detect additional food relative to the prey. Thus, the term $\xi=\eta A$ represents quantity of additional food perceptible to predator relative to the prey. $a_{1}=\frac{a}{h_{N} e_{N}}$ is the normalisation coefficient that relates the densities of predator and prey. $c_{1}=\frac{c}{h_{N}}$ stands for maximum rate of predation. If $\epsilon \in(0,1)$ represents the efficiency with which the food consumed by the predator gets converted into predator biomass then the maximum growth rate of the predator given by $\epsilon c_{1}$ is represented by the parameter $b_{1}$, and therefore, the growth rate of predator $P$ is of the form $f(N)=\frac{b_{1}(N+\xi)}{a_{1}+\alpha \xi+N}$. Incorporating the above facts, Kumar and Chakrabarty [16], Srinivasu et al. [26], Sahoo and Poria [24] and Ghorai and Poria [10] proposed and analyzed the following predator-prey system with additional food

$$
\left\{\begin{array}{l}
\frac{\mathrm{d} N(t)}{\mathrm{d} t}=r N(t)\left(1-\frac{N(t)}{K}\right)-f(N(t), P(t)), \\
\frac{\mathrm{d} P(t)}{\mathrm{d} t}=\mu f(N(t), P(t))-l P(t)
\end{array}\right.
$$

where $f(N, P)$ is of the form (a) Holling type-II: $f(N, P)=\frac{c_{1}(N+\xi) P}{a_{1}+\alpha \xi+N}$; (b) Holling type-III: $f(N, P)=$ $\frac{c_{1}\left(N^{2}+\xi^{2}\right) P}{a_{1}+\alpha \xi^{2}+N^{2}}$; (c) Ratio-dependent type: $f(N, P)=\frac{c_{1}(N+\xi) P}{N+P+\alpha \xi}$; (d) Beddington-DeAngelis: $f(N, P)=\frac{c_{1}(N+\xi) P}{a_{1}+N+\alpha \xi+\rho P}$, respectively. In a real ecosystem, population is always subjected to random disturbances. May [22] pointed out that continuous fluctuation in the environment can lead to random fluctuation in the birth rates, death rates, carrying capacity, competition coefficients and all other parameters involved in a dynamical model, to a great lesser extent. Recently predator-prey models in random environment have also gained much attention $[1,4,7,12,18,30]$. Das and Samanta [8] established a stochastic prey-predator model with additional food for predator. They studied the persistence of the system under obtained conditions and how the solution of the underlying system is globally attractive in mean. Besides white noise, population may be disturbed by telephone noise which makes population system switch from one regime to another, the stochastic differential equations driven by a continuous-time Markov chain have been used to model the population systems [3]. Based on the above discussion, we present the following system:

$$
\left\{\begin{aligned}
\mathrm{d} N(t)= & N(t)\left[r\left(\Lambda_{t}\right)\left(1-\frac{N(t)}{K}\right)-\frac{c_{1}\left(\Lambda_{t}\right) P(t)}{a_{1}\left(\Lambda_{t}\right)+\alpha\left(\Lambda_{t}\right) \xi\left(\Lambda_{t}\right)+N(t)}\right] \mathrm{d} t \\
& +N(t)\left[\sigma_{11}\left(\Lambda_{t}\right)+\sigma_{12}\left(\Lambda_{t}\right) N(t)\right] \mathrm{d} B_{1}(t) \\
\mathrm{d} P(t)= & P(t)\left(\frac{b_{1}\left(\Lambda_{t}\right)\left(N(t)+\xi\left(\Lambda_{t}\right)\right)}{a_{1}\left(\Lambda_{t}\right)+\alpha\left(\Lambda_{t}\right) \xi\left(\Lambda_{t}\right)+N(t)}-l\left(\Lambda_{t}\right)\right) \mathrm{d} t \\
& +P(t)\left[\sigma_{21}\left(\Lambda_{t}\right)+\sigma_{22}\left(\Lambda_{t}\right) P(t)\right] \mathrm{d} B_{2}(t),
\end{aligned}\right.
$$


where $B_{1}(t), B_{2}(t)$ are mutually independent Browian motions defined on the complete probability space $\left(\Omega, \mathcal{F},\left\{\mathcal{F}_{t}\right\}_{t \geq 0}, \mathbb{P}\right)$ and nonnegative constants $\sigma_{i j}$ denote the intensities of the white noise. $\Lambda_{t}$ is a continuous time Markov chain with a finite state space $\mathcal{M}=\{1,2, \ldots, n\}, 1 \leq n<\infty$. Obviously, assuming no Markov chain $\Lambda_{t}$, if $\sigma_{i 2}=0(i=1,2)$, our model becomes the system in [8]; if $\sigma_{i j}=0(i, j=1,2)$, our model becomes the system (1.1) with Holling type-II response. It should be noted that the present model is made more realistic by considering noises and predator-prey interference. But there is no paper about the ergodic property of model (1.2). So the contribution of current work are as follows:

(i) we established the condition for extinction of stochastic predator-prey model with Markov switching.

(ii) We obtain the ergodic property and positive recurrence which implies the existence and uniqueness of a stationary distribution.

The organizations of the paper are as follows. Preliminary results are provided in Section 2. In Section 3, the conditions for extinction of prey and predator are discussed. In Section 4, we discuss the sufficient conditions for the existence of a unique ergodic stationary distribution to system (1.2). Section 5 illustrates our results through numerical simulations. Finally, conclusion and discussion are given to end this paper.

\section{Preliminaries}

In this section, we introduce the notations and lemmas which will be used in the whole paper. Let $(\Omega, \mathcal{F}, \mathbb{P})$ be a complete probability space with a filtration $\left\{\mathcal{F}_{t}\right\}_{t>0}$ satisfying the usual conditions (i.e., it is increasing and right continuous while $\mathcal{F}_{0}$ contains all $\mathbb{P}$ - null sets). $\mathbb{R}_{+}^{2}$ denotes the positive zone in $\mathbb{R}^{n}$, i.e., $\mathbb{R}_{+}^{2}=\left\{\left(x_{1}, x_{2}\right) \in\right.$ $\left.\mathbb{R}^{2}, x_{i}>0, i=1,2\right\}$. Let $\Lambda(t)$ be a right-continuous Markov chain on the probability space taking values in a finite state space $\mathcal{M}=\{1,2, \ldots, n\}$ with generator $\Gamma=\left(\gamma_{i j}\right)_{N \times N}$ given by

$$
P\{\Lambda(t+\delta)=j \mid \Lambda(t)=i\}=\left\{\begin{array}{cc}
\gamma_{i j} \delta+o(\delta), & \text { if } \quad i \neq j \\
1+\gamma_{i j} \delta+o(\delta), & \text { if } \quad i=j
\end{array}\right.
$$

where $\delta>0$. Here $\gamma_{i j} \geq 0$ is the transition rate from $i$ to $j$ if $i \neq j$, while $\sum_{j=1}^{n} \gamma_{i j}=0$. Assume further that the Markov chain $\Lambda(t)$ is irreducible and has a unique stationary distribution $\pi=\left(\pi_{1}, \pi_{2}, \ldots, \pi_{n}\right)$ which can be determined by equation

$$
\pi \Gamma=0,
$$

subject to

$$
\sum_{h=1}^{n} \pi_{h}=1, \quad \pi_{h}>0, \quad \forall h \in \mathcal{M}
$$

We assume that the Markov chain $\Lambda(t)$ is independent of the Brownian motion $B(t)$. For any vector $g=$ $(g(1), g(2), \ldots, g(n))$, define $\hat{g}=\min _{k \in \mathcal{M}} g(k), \check{g}=\max _{k \in \mathcal{M}} g(k)$.

Lemma 2.1. ([29]) The irreducible generator $\Gamma$ has a unique stationary distribution $\pi=\left(\pi_{1}, \pi_{2}, \ldots, \pi_{n}\right)$ satisfying $\pi_{i}>0$, then the equation $\Gamma c=\eta$ has a solution if and only if $\pi \eta=0$, where $\pi$ and $\eta \in \mathbb{R}^{n}$.

Let $(X(t), \Lambda(t))$ is the diffusion Markov process and satisfy the following equation

$$
\mathrm{d} X(t)=b(X(t), \Lambda(t)) d t+\sigma(X(t), \Lambda(t)) d B(t), \quad X(0)=x_{0}, \quad \Lambda(0)=r_{0},
$$

where $b(\cdot, \cdot): \mathbb{R}^{n} \times \mathcal{M} \rightarrow \mathbb{R}^{n}, \sigma(\cdot, \cdot): \mathbb{R}^{n} \times \mathcal{M} \rightarrow \mathbb{R}^{n \times n}$, and $D(X, k)=\sigma(X, k) \sigma^{T}(X, k)=\left(d_{i j}(X, k)\right)$. For each $k \in \mathcal{M}$, let $V(\cdot, k)$ be any twice continuously differentiable function, the differential operator $L$ of $(2.2)$ is defined 
by

$$
L V(X, k)=\sum_{i=1}^{n} b_{i}(X, k) \frac{\partial V(X, k)}{\partial X_{i}}+\frac{1}{2} \sum_{i=1}^{n} d_{i j}(X, k) \frac{\partial^{2} V(X, k)}{\partial X_{i} \partial X_{j}}+\Gamma V(X, \cdot)(k)
$$

where $\Gamma V(X, \cdot)(k)=\sum_{l=1}^{n} \gamma_{k l} V(X, \cdot)(l)$. Denote the solution of $(2.2)$ by $\left(X^{x_{0}, r_{0}}, \Lambda(t)\right)$ if the emphasis on the initial data is needed.

Definition 2.2. For $\mathcal{U}:=\mathcal{D} \times J$, where $J \subset \mathcal{M}$ and $\mathcal{D} \subset \mathbb{R}^{2}$ is an open set with compact closure. Let $\tau_{\mathcal{U}}=\inf \left\{t>0 ;\left(X^{x_{0}, r_{0}}(t), \Lambda(t)\right) \in \mathcal{U}\right\}$. A Markov process $\left(X^{x_{0}, r_{0}}(t), \Lambda(t)\right)$ defined on $\mathbb{R}^{n} \times \mathcal{M}$ is said to be recurrent with respect to some bounded set $\mathcal{U}$. if $\mathcal{P}_{x_{0}, r_{0}}\left(\tau_{\mathcal{U}}<\infty\right)=1$ for any $\left(x_{0}, r_{0}\right) \in \mathcal{D}^{c} \times \mathcal{M}$. A recurrent process $\left(X^{x_{0}, r_{0}}(t), \Lambda(t)\right)$ with finite mean recurrence time is said to be positive recurrent with respect to $\mathcal{U}$, i.e., $\mathbb{E}_{x_{0}, r_{0}}\left(\tau_{\mathcal{U}}\right)<\infty$.

Lemma 2.3. ([13]) There exists a bounded domain $\mathcal{U}:=\mathcal{D} \times \mathcal{M}$, having the properties that:

(i) In the domain $\mathcal{D}$ and some neighborhood thereof, the smallest eigenvalue of the diffusion matrix $D(x, k)$ is bounded away from zero for each $k \in \mathcal{M}$.

(ii) If $\left(x_{0}, r_{0}\right) \in \mathcal{D}^{c} \times \mathcal{M}$, the mean time $\tau_{\mathcal{U}}$ at which a path issuing from $\left(x_{0}, r_{0}\right)$ reaches the set $\mathcal{U}$ is finite, and $\sup _{\left(x_{0}, r_{0}\right) \in \mathcal{K} \times \mathcal{M}} \mathbb{E}_{x_{0}, r_{0}} \tau_{\mathcal{U}}<\infty$ for every compact subset $\mathcal{K} \in \mathbb{R}^{2}$. Then the Markov process $(X(t), \Lambda(t))$ has a unique stationary distribution $\nu(\cdot)$. Furthermore, for any Borel measurable function $f$ such that

$$
\sum_{i=1}^{N} \int_{\mathbb{R}^{2}} f(y, i) \mathrm{d} \nu(y, i)<\infty
$$

we have

$$
P\left(\lim _{t \rightarrow \infty} \frac{1}{t} \int_{0}^{t} f(z(s), \Lambda(s)) \mathrm{d} s=\sum_{i=1}^{N} \int_{\mathbb{R}^{2}} f(y, i) \mathrm{d} \nu(y, i)\right)=1
$$

To validate conditions (i) and (ii), it suffices to prove that for each $k \in \mathcal{M}, D(x, k)$ is symmetric and satisfies $\varrho|\xi|^{2} \leq \xi^{T} D(x, k) \xi \leq \varrho^{-1}|\xi|^{2}$ for all $\xi \in \mathbb{R}^{2}$, and there exist a domain $\mathcal{D} \subset \mathbb{R}^{2}$ with compact closure satisfying that, for each $k \in \mathcal{M}$, there exists a twice continuously differentiable nonnegative function $V(\cdot, i): \mathcal{D}^{c} \rightarrow \mathbb{R}_{+}$ such that for some $\zeta>0, L V(X, i)<-\zeta[28]$.

The following theorem concerns the existence and uniqueness of positive solutions. Since the proof is standard (see $[17,30]$ ), we only present it without proof.

Theorem 2.4. For any initial value $(N(0), P(0), \Lambda(0)) \in \mathbb{R}_{+}^{2} \times \mathcal{M}$, there exists a unique solution to system (1.2) on $t \geq 0$ and the solution will remain in $\mathbb{R}_{+}^{2} \times \mathcal{M}$ with probability 1.

\section{ExtinCtion.}

In the study of population dynamics, we are interested in whether a population can sustain development or become extinct. Let us first define local extinction of a population.

Definition 3.1. The predator population is said to be extinct almost surely, if $\lim _{t \rightarrow \infty} P(t)=0$ a.s., the prey population is said to be extinct almost surely, if $\lim _{t \rightarrow \infty} N(t)=0$ a.s. 
Theorem 3.2. Let $(N(t), P(t), \Lambda(t))$ be defined by (1.2) and $\pi$ be the stationary distribution of the process $\Lambda(t)$.

(i) If $\sum_{i \in \mathcal{M}} \pi_{i}\left(r(i)-\frac{\sigma_{11}^{2}(i)}{2}\right)<0$, then the species $N$ will become extinct a.s., i.e., $\lim _{t \rightarrow \infty} N(t)=0$ a.s. Moreover, if $\sum_{i \in \mathcal{M}} \pi_{i}\left(\frac{b_{1}(i) \xi(i)}{a_{1}(i)+\alpha(i) \xi(i)}\right)-l(i)-\frac{\sigma_{21}^{2}(i)}{2}<0$, then the species $P$ will become extinct a.s., i.e., $\lim _{t \rightarrow \infty} P(t)=0$ a.s.

(ii) If $\sum_{i \in \mathcal{M}} \pi_{i}\left(b(i)+\frac{b_{1}(i) \xi(i)}{a_{1}(i)+\alpha(i) \xi(i)}-l(i)-\frac{\sigma_{21}^{2}(i)}{2}\right)<0$, then the species $P$ will become extinct a.s., i.e., $\lim _{t \rightarrow \infty} P(t)=0$ a.s.

Proof. Applying the Itô's formula to the first and second equation of system (1.2) respectively leads to

$$
\begin{aligned}
\mathrm{d} \ln N(t)= & {\left[r\left(\Lambda_{t}\right)\left(1-\frac{N(t)}{K}\right)-\frac{c_{1}\left(\Lambda_{t}\right) P(t)}{a_{1}\left(\Lambda_{t}\right)+\alpha\left(\Lambda_{t}\right) \xi\left(\Lambda_{t}\right)+N(t)}-\frac{\sigma_{11}^{2}\left(\Lambda_{t}\right)}{2}-\sigma_{11}\left(\Lambda_{t}\right) \sigma_{12}\left(\Lambda_{t}\right) N(t)\right.} \\
& \left.-\frac{\sigma_{12}^{2}\left(\Lambda_{t}\right)}{2} N^{2}(t)\right] \mathrm{d} t+\sigma_{11}\left(\Lambda_{t}\right) \mathrm{d} B_{1}(t)+\sigma_{12}\left(\Lambda_{t}\right) N(t) \mathrm{d} B_{1}(t) \\
\leq & \left(r\left(\Lambda_{t}\right)-\frac{\sigma_{11}^{2}\left(\Lambda_{t}\right)}{2}-\frac{\sigma_{12}^{2}\left(\Lambda_{t}\right)}{2} N^{2}(t)\right) \mathrm{d} t+\sigma_{11}\left(\Lambda_{t}\right) \mathrm{d} B_{1}(t)+\sigma_{12}\left(\Lambda_{t}\right) N(t) \mathrm{d} B_{1}(t),
\end{aligned}
$$

and

$$
\begin{aligned}
\mathrm{d} \ln P(t)= & {\left[\frac{b_{1}\left(\Lambda_{t}\right)\left(N(t)+\xi\left(\Lambda_{t}\right)\right)}{a_{1}\left(\Lambda_{t}\right)+\alpha\left(\Lambda_{t}\right) \xi\left(\Lambda_{t}\right)+N(t)}-l\left(\Lambda_{t}\right)-\frac{\sigma_{21}^{2}\left(\Lambda_{t}\right)}{2}-\sigma_{21}\left(\Lambda_{t}\right) \sigma_{22}\left(\Lambda_{t}\right) P(t)-\frac{\sigma_{22}^{2}\left(\Lambda_{t}\right)}{2} P^{2}(t)\right] \mathrm{d} t } \\
& +\sigma_{21}\left(\Lambda_{t}\right) \mathrm{d} B_{2}(t)+\sigma_{22}\left(\Lambda_{t}\right) P(t) \mathrm{d} B_{2}(t) \\
\leq \quad & {\left[\frac{b_{1}\left(\Lambda_{t}\right) N(t)}{a_{1}\left(\Lambda_{t}\right)+\alpha\left(\Lambda_{t}\right) \xi\left(\Lambda_{t}\right)+N(t)}-\left(l\left(\Lambda_{t}\right)+\frac{\sigma_{21}^{2}\left(\Lambda_{t}\right)}{2}-\frac{b_{1}\left(\Lambda_{t}\right) \xi\left(\Lambda_{t}\right)}{a_{1}\left(\Lambda_{t}\right)+\alpha\left(\Lambda_{t}\right) \xi\left(\Lambda_{t}\right)}\right)-\frac{\sigma_{22}^{2}\left(\Lambda_{t}\right)}{2} P^{2}(t)\right] \mathrm{d} t } \\
& +\sigma_{21}\left(\Lambda_{t}\right) \mathrm{d} B_{2}(t)+\sigma_{22}\left(\Lambda_{t}\right) P(t) \mathrm{d} B_{2}(t) .
\end{aligned}
$$

Integrating both sides of (3.1) and (3.2) from 0 to $t$, we obtain

$$
\ln N(t) \leq \ln N(0)+\int_{0}^{t}\left(r\left(\Lambda_{s}\right)-\frac{\sigma_{11}^{2}\left(\Lambda_{s}\right)}{2}\right) \mathrm{d} s+\int_{0}^{t} \sigma_{11}\left(\Lambda_{s}\right) \mathrm{d} B_{1}(s)-\frac{1}{2}\left\langle M_{1}, M_{1}\right\rangle(t)+M_{1}(t)
$$

and

$$
\begin{aligned}
\ln P(t) \leq & \ln P(0)+\int_{0}^{t} \frac{b_{1}\left(\Lambda_{s}\right) N(s)}{a_{1}\left(\Lambda_{s}\right)+\alpha\left(\Lambda_{s}\right) \xi\left(\Lambda_{s}\right)+N(s)} \mathrm{d} s+\int_{0}^{t} \sigma_{21}\left(\Lambda_{s}\right) \mathrm{d} B_{2}(s) \\
& -\int_{0}^{t}\left(l\left(\Lambda_{s}\right)+\frac{\sigma_{21}^{2}\left(\Lambda_{s}\right)}{2}-\frac{b_{1}\left(\Lambda_{s}\right) \xi\left(\Lambda_{s}\right)}{a_{1}\left(\Lambda_{s}\right)+\alpha\left(\Lambda_{s}\right) \xi\left(\Lambda_{s}\right)}\right) \mathrm{d} s-\frac{1}{2}\left\langle M_{2}, M_{2}\right\rangle(t)+M_{2}(t),
\end{aligned}
$$

where $M_{1}(t)=\int_{0}^{t} \sigma_{12}\left(\Lambda_{s}\right) N(s) \mathrm{d} B_{1}(s)$ and $M_{2}(t)=\int_{0}^{t} \sigma_{22}\left(\Lambda_{s}\right) P(s) \mathrm{d} B_{2}(s)$, whose quadratic variations are

$$
\left\langle M_{1}, M_{1}\right\rangle(t)=\int_{0}^{t} \sigma_{12}^{2}\left(\Lambda_{s}\right) N^{2}(s) \mathrm{d} s \quad \text { and } \quad\left\langle M_{2}, M_{2}\right\rangle(t)=\int_{0}^{t} \sigma_{22}^{2}\left(\Lambda_{s}\right) P^{2}(s) \mathrm{d} s,
$$


respectively. Using the exponential martingales inequality [21], we get

$$
\mathbb{P}\left\{\sup _{0 \leq t \leq \varrho}\left[M_{i}(t)-\frac{1}{2}\left\langle M_{i}, M_{i}\right\rangle(t)\right]>2 \ln \varrho\right\} \leq \frac{1}{\varrho^{2}}, i=1,2 .
$$

By the Borel-Cantelli Lemma [21], one can see that for almost all $\omega \in \Omega$, there is a random integer $\varrho_{0}=\varrho_{0}(\omega)$ such that for $\varrho \geq \varrho_{0}$,

$$
\sup _{0 \leq t \leq n}\left[M_{i}(t)-\frac{1}{2}\left\langle M_{i}, M_{i}\right\rangle(t)\right] \leq 2 \ln \varrho, i=1,2
$$

Substituting the inequality into (3.1) and (3.2) gives

$$
\begin{aligned}
\frac{\ln N(t)}{t} & \leq \frac{\ln N(0)}{t}+\frac{1}{t} \int_{0}^{t}\left(r\left(\Lambda_{s}\right)-\frac{\sigma_{11}^{2}\left(\Lambda_{s}\right)}{2}\right) \mathrm{d} s+\frac{1}{t} \int_{0}^{t} \sigma_{11}\left(\Lambda_{s}\right) \mathrm{d} B_{1}(s)+\frac{2 \ln \varrho}{t} \\
& =: \frac{\ln \varphi(t)}{t}+\frac{2 \ln \varrho}{t}
\end{aligned}
$$

and

$$
\begin{aligned}
\frac{\ln P(t)}{t} \leq & \frac{\ln P(0)}{t}+\frac{1}{t} \int_{0}^{t} \frac{b_{1}\left(\Lambda_{s}\right) N(s)}{a_{1}\left(\Lambda_{s}\right)+\alpha\left(\Lambda_{s}\right) \xi\left(\Lambda_{s}\right)+N(s)} \mathrm{d} s+\frac{1}{t} \int_{0}^{t} \sigma_{21}\left(\Lambda_{s}\right) \mathrm{d} B_{2}(s) \\
& -\frac{1}{t} \int_{0}^{t}\left(l\left(\Lambda_{s}\right)+\frac{\sigma_{21}^{2}\left(\Lambda_{s}\right)}{2}-\frac{b_{1}\left(\Lambda_{s}\right) \xi\left(\Lambda_{s}\right)}{a_{1}\left(\Lambda_{s}\right)+\alpha\left(\Lambda_{s}\right) \xi\left(\Lambda_{s}\right)}\right) \mathrm{d} s+\frac{2 \ln \varrho}{t}
\end{aligned}
$$

for all $0 \leq t \leq \varrho, \varrho \geq \varrho_{0}$ a.s. By the strong egodicity theorem,

$$
\lim _{t \rightarrow \infty}\left\{\frac{1}{t} \int_{0}^{t}\left(r\left(\Lambda_{s}\right)-\frac{1}{2} \sigma_{11}^{2}\left(\Lambda_{s}\right)\right) \mathrm{d} s+\frac{1}{t} \int_{0}^{t} \sigma_{11}\left(\Lambda_{s}\right) \mathrm{d} B_{1}(s)\right\}=\sum_{i \in \mathcal{M}} \pi_{i}\left(r(i)-\frac{\sigma_{11}^{2}(i)}{2}\right) \text { a.s. }
$$

Here we have used the fact

$$
\lim _{t \rightarrow \infty} \frac{1}{t} \int_{0}^{t} \sigma_{11}\left(\Lambda_{s}\right) \mathrm{d} B_{1}(s)=0 \text { a.s. }
$$

which is due to the boundedness of $\sigma_{11}(k)$ and the strong law of large numbers. It then follows from (3.5) that

$$
\limsup _{t \rightarrow \infty} \frac{\ln N(t)}{t} \leq \lim _{t \rightarrow \infty}\left\{\frac{\ln \varphi(t)}{t}+\frac{2 \ln \nu}{t}\right\}=: \sum_{i \in \mathcal{M}} \pi_{i}\left(r(i)-\frac{\sigma_{11}^{2}(i)}{2}\right) \text { a.s. }
$$

Therefore, when $\sum_{i \in \mathcal{M}} \pi_{i}\left(r(i)-\frac{\sigma_{11}^{2}(i)}{2}\right)<0$, we have $\limsup _{t \rightarrow \infty} \frac{\ln N(t)}{t}<0$ a.s., which further implies that $\lim _{t \rightarrow \infty} N(t)=0$ a.s. Thus for any small $\epsilon>0$ there exists a $t_{0}$ and a set $\Omega_{\epsilon} \subset \Omega$ such that $P\left(\Omega_{\epsilon}\right)>1-\epsilon$ and $\frac{b_{1}\left(\Lambda_{t}\right) N(t)}{a_{1}\left(\Lambda_{t}\right)+\alpha\left(\Lambda_{t}\right) \xi\left(\Lambda_{t}\right)+N(t)}<\epsilon$ a.s. for $t \geq t_{0}$ and $\omega \in \Omega_{\epsilon}$. Furthermore, by making use of the ergodicity of Markovian switching, we have

$$
\limsup _{t \rightarrow \infty} \frac{\ln P(t)}{t} \leq \sum_{i \in \mathcal{M}} \pi_{i}\left(\frac{b_{1}(i) \xi(i)}{a_{1}(i)+\alpha(i) \xi(i)}-l(i)-\frac{\sigma_{21}^{2}(i)}{2}\right) \mathrm{d} s .
$$


One can conclude that if $\sum_{i \in \mathcal{M}} \pi_{i}\left(\frac{b_{1}(i) \xi(i)}{a_{1}(i)+\alpha(i) \xi(i)}-l(i)-\frac{\sigma_{21}^{2}(i)}{2}\right)<0$, then $\limsup _{t \rightarrow \infty} \frac{\ln P(t)}{t}<0$. Thus, $\lim _{t \rightarrow \infty} P(t)=0$ a.s. This completes the proof of (i).

Next, we verify (ii) of Theorem 3.2. Recall (3.6) we obtain

$$
\begin{aligned}
\frac{\ln P(t)}{t} \leq & \frac{\ln P(0)}{t}+\frac{1}{t} \int_{0}^{t}\left(b_{1}\left(\Lambda_{s}\right)+\frac{b_{1}\left(\Lambda_{s}\right) \xi\left(\Lambda_{s}\right)}{a_{1}+\alpha\left(\Lambda_{s}\right) \xi\left(\Lambda_{s}\right)}-l(i)-\frac{\sigma_{21}^{2}\left(\Lambda_{s}\right)}{2}\right) \mathrm{d} s \\
& +\frac{1}{t} \int_{0}^{t} \sigma_{21}\left(\Lambda_{s}\right) \mathrm{d} B_{2}(s)+\frac{2 \ln \varrho}{t} .
\end{aligned}
$$

Take the limits of both sides, we have

$$
\limsup t \rightarrow \infty \frac{\ln P(t)}{t} \leq \sum_{i \in \mathcal{M}} \pi_{i}\left(b_{1}(i)+\frac{b_{1}(i) \xi(i)}{a_{1}(i)+\alpha(i) \xi(i)}-l(i)-\frac{\sigma_{21}^{2}(i)}{2}\right) \text { a.s. }
$$

We can conclude that if $\sum_{i \in \mathcal{M}} \pi_{i}\left(b_{1}(i)+\frac{b_{1}(i) \xi(i)}{a_{1}(i)+\alpha(i) \xi(i)}-l(i)-\frac{\sigma_{21}^{2}(i)}{2}\right)<0$, then $\lim _{t \rightarrow \infty} P(t)=0$ a.s. This completes the proof of (ii).

From the first result of this theorem it can be concluded that the large intensity noise may be a cause for extinction of the prey population, which means that unpredictable events in nature can be fetal for prey population. In the second result of this theorem it was proved that if the intensity of the noise is sufficiently large, then predator population becomes extinct regardless of the size of prey population. If there is no Markovian switching, our results are consistent to Theorems 4.1 and 4.2 in [28]. Hence, Theorem 1 generalizes Theorems 4.1 and 4.2 in [28].

\section{EXISTENCE OF ERGODIC STATIONARY DISTRIBUTION}

In this section, we will establish sufficient conditions for positive recurrence and the existence of an ergodic stationary distribution of system (1.2). The existence of an ergodic stationary distribution in the context of predator-prey systems mean that the predator and prey populations can be persistent in the long time. First of all, we give the following lemma.

Lemma 4.1. The following equation

$$
-\frac{b_{1}(k)\left(a_{1}(k)+\alpha(k) \xi(k)-\xi(k)\right)}{\left(a_{1}(k)+\alpha(k) \xi(k)+K\right)^{2}}+d_{1}(k) r(k)-\sum_{j \in \mathcal{M}} \gamma_{k j} d_{1}(j)=0, \quad k=1,2, \cdots, N .
$$

has a solution $\left(d_{1}(1), d_{1}(2), \ldots, d_{1}(n)\right)^{T}$

Proof. The equation (4.1) can be rewritten as the following form

$$
A x=B,
$$


where $x \in \mathbb{R}^{n}, B=\left(\frac{b_{1}(1)\left(a_{1}(1)+\alpha(1) \xi(1)-\xi(1)\right)}{\left(a_{1}(1)+\alpha(1) \xi(1)+K\right)^{2}}, \frac{b_{1}(2)\left(a_{1}(2)+\alpha(2) \xi(2)-\xi(2)\right)}{\left(a_{1}(2)+\alpha(2) \xi(2)+K\right)^{2}}, \ldots, \frac{b_{1}(n)\left(a_{1}(n)+\alpha(n) \xi(n)-\xi(n)\right)}{\left(a_{1}(n)+\alpha(n) \xi(n)+K\right)^{2}}\right)^{T}$ is an $n \times$ 1-dimensional vector and

$$
A=\left[\begin{array}{cccc}
r(1)-\gamma_{11} & -\gamma_{12} & \cdots & -\gamma_{1 n} \\
-\gamma_{21} & r(2)-\gamma_{22} & \cdots & -\gamma_{2 n} \\
\vdots & \vdots & \vdots & \vdots \\
-\gamma_{n 1} & -\gamma_{n 2} & \cdots & r(n)-\gamma_{n n}
\end{array}\right]
$$

Clearly, $A \in \mathbb{Z}_{n \times n}$. The matrix $A$ can be expressed in the form $A=\operatorname{diag}(r(k)) I-\Gamma, k=1, \ldots, n$, where $I$ is the identity $n \times n$ matrix and $\Gamma$ is an irreducible matrix. So we obtain $\operatorname{diag}\left(\frac{1}{r(k)}\right) A=I-\tilde{\Gamma}, \tilde{\Gamma}=\operatorname{diag}\left(\frac{1}{r(k)}\right) \Gamma$. Obviously, $\operatorname{diag}\left(\frac{1}{r(k)}\right) A$ is a nonsingular $M$-matrix which leads to that $A$ is also a nonsingular $M$-matrix. Therefore, for the vector $B \in \mathbb{R}^{n}$, the linear equation $(4.1)$ has a solution $\left(d_{1}(1), \ldots, d_{1}(n)\right)^{T}$. This completes the proof.

Let us take a constant $d_{2}>\max \left\{0, \max _{k \in \mathcal{M}}\left\{\frac{\frac{2 K b_{1}(k)}{K\left(a_{1}(k)+\alpha(k) \xi(k)\right)^{2}}+d_{1}(k) r(k)}{r(k)-2 K^{2} \sigma_{12}^{2}(k)}\right\}\right\}$ such that

$$
\lambda=\sum_{k \in \mathcal{M}} \pi_{k} \lambda_{k}>0
$$

where $\lambda_{k}=-\frac{b_{1}(k)(K+\xi(k))}{a_{1}(k)+\alpha(k) \xi(k)+K}+l(k)+\sigma_{21}^{2}(k)+d_{2} K \sigma_{11}^{2}(k)+2 d_{2} K^{3} \sigma_{12}^{2}(k)$.

Theorem 4.2. If $\hat{l}>\frac{\breve{b}_{1} \check{\xi}}{\hat{a}_{1}+\hat{\alpha} \hat{\xi}}, \min _{k \in \mathcal{M}}\left\{r(k)-2 K^{2} \sigma_{12}^{2}(k)\right\}>0$, then there exists a positive solution $(N(t), P(t), \Lambda(t))$ of system (1.2) which is positive recurrent and has a unique ergodic stationary distribution.

Proof. In order to proof Theorem 4.2, it suffices to verify the condition in Lemma 2.3 Denote the constant $0<v<1$ and a positive constant $M=\frac{4}{\lambda} \max \left\{2, \sup _{(N, P) \in \mathbb{R}_{+}^{2}}\left\{-\frac{1-v}{4}\left(\hat{\sigma}_{12} N^{v+2}+\hat{\sigma}_{22} P^{v+2}\right)+\check{r} N^{v}\right\}\right\}$ (see the proof below ). Define a $C^{2}$-function $\widetilde{V}: \mathbb{R}_{+}^{2} \times \mathcal{M} \rightarrow \mathbb{R}$ in the following form

$$
\tilde{V}(N, P, k)=M\left[-\ln P-d_{1}(k) N+d_{2}\left(N-K-K \ln \frac{N}{K}\right)+\frac{d_{3}}{v} P^{v}+d_{4} P\right]+\frac{1}{v}\left(N^{v}+P^{v}\right),
$$

where $d_{1}(k)$ is the solution of $(4.1), d_{2}\left(d_{2}>d_{1}(k)\right.$, for each $\left.k \in \mathcal{M}\right)$ satisfies the equation $(4.2)$ and $d_{3}$ is a positive constant to be determined later, $d_{4}=\frac{\check{d}_{2} K \check{c}_{1}(1-\hat{m})}{\hat{l}\left(\hat{a}_{1}+\hat{\alpha} \hat{\xi}\right)-\breve{b}_{1} \tilde{\xi}}$. It is not difficult to check that there is a unique minimum value point $\widetilde{V}\left(N_{0}(k), P_{0}(k), k\right)$ of $\widetilde{V}(N, P, k)$. In fact, functions like $a x-b-c \ln x$ are monotonically increasing and have minimal values, where $a, b, c$ are positive constants. Thus $\tilde{V}(N, P, k)$ is a nonnegative function with a minimum $\tilde{V}\left(N_{0}(k), P_{0}(k), k\right)$.

Define a $C^{2}$-function $V: \mathbb{R}_{+}^{2} \rightarrow \mathbb{R}$ by

$$
\begin{aligned}
V(N, P, k)= & \tilde{V}(N, P, k)-\tilde{V}\left(N_{0}(k), P_{0}(k), k\right)+M\left(\omega_{k}+\left|\omega_{k}\right|\right) \\
= & M\left[-\ln P-d_{1}(k) N+d_{2}\left(N-K-K \ln \frac{N}{K}\right)+\frac{d_{3}}{v} p^{v}+d_{4} P\right]+\frac{1}{v}\left(N^{v}+P^{v}\right) \\
& +M\left(\omega_{k}+\left|\omega_{k}\right|\right)-\tilde{V}\left(N_{0}(k), P_{0}(k), k\right),
\end{aligned}
$$


where $\omega=\left(\omega_{1}, \omega_{2}, \ldots, \omega_{n}\right)^{T},|\omega|=\sqrt{\sum_{i=1}^{n} \omega_{i}^{2}}$ and $\omega_{k}(k \in \mathcal{M})$ will be determined later. Here $|\omega|$ is used to guarantee that $\omega_{k}+\left|\omega_{k}\right|$ is nonnegative. So the $C^{2}$-function $V(N, P, k)$ is nonnegative. Denote by $V_{1}=$ $-\ln P-d_{1}(k) N+d_{2}\left(N-K-K \ln \frac{N}{K}\right)+\left(\omega_{k}+\left|\omega_{k}\right|\right), V_{2}=\frac{d_{3}}{v} P^{v}, V_{3}=d_{4} P, V_{4}=\frac{1}{v}\left(N^{v}+P^{v}\right)$. By use of the generalized Itô's formula to $V_{1}$, we obtain

$$
\begin{aligned}
L V_{1}= & -\frac{b_{1}(k)(N+\xi(k))}{a_{1}(k)+\alpha(k) \xi(k)+N}+l(k)+\frac{1}{2} \sigma_{21}^{2}(k)+\sigma_{21}(k) \sigma_{22}(k) P+\frac{1}{2} \sigma_{22}^{2}(k) P^{2} \\
& +\frac{d_{1}(k) r(k)}{K} N(N-K)+\frac{d_{1}(k) c_{1}(k) N P}{a_{1}(k)+\alpha(k) \xi(k)+N}-\frac{d_{2} r(k)}{K}(N-K)^{2} \\
& -\frac{d_{2} c_{1}(k) N P}{a_{1}(k)+\alpha(k) \xi(k)+N}+\frac{d_{2} K c_{1}(k)(1-m(k)) P}{a_{1}(k)+\alpha(k) \xi(k)+N}-\sum_{j \in \mathcal{M}} \gamma_{k j} d_{1}(j)(N-K+K) \\
& +\frac{d_{2} K}{2} \sigma_{11}^{2}(k)+d_{2} K \sigma_{11}(k) \sigma_{12}(k) N+\frac{d_{2} K}{2} \sigma_{12}^{2}(k) N^{2}+\sum_{j \neq k \in \mathcal{M}} \gamma_{k j}\left(\omega_{j}-\omega_{k}\right) \\
\leq & -\frac{b_{1}(k)(N+\xi(k))}{a_{1}(k)+\alpha(k) \xi(k)+N}+l(k)+\frac{1}{2} \sigma_{21}^{2}(k)+\frac{1}{2} \sigma_{21}^{2}(k)+\frac{1}{2} \sigma_{22}^{2}(k) P^{2}+\frac{1}{2} \sigma_{22}^{2}(k) P^{2} \\
& +\frac{d_{1}(k) r(k)}{K} N(N-K)+\frac{d_{1}(k) c_{1}(k) N P}{a_{1}(k)+\alpha(k) \xi(k)+N}-\frac{d_{2} r(k)}{K}(N-K)^{2} \\
& -\frac{b_{1}(k)(K+\xi(k))}{a_{1}(k)+\alpha(k) \xi(k)+K}+\frac{b_{1}(k)(K+\xi(k))}{a_{1}(k)+\alpha(k) \xi(k)+K}+\sum_{j \neq k \in \mathcal{M}} \gamma_{k j}\left(\omega_{j}-\omega_{k}\right) \\
& -\frac{d_{2} c_{1}(k) N P}{a_{1}(k)+\alpha(k) \xi(k)+N}+\frac{d_{2} K c_{1}(k) P}{a_{1}(k)+\alpha(k) \xi(k)+N}-\sum_{j \in \mathcal{M}} \gamma_{k j} d_{1}(j)(N-K+K) \\
& +\frac{d_{2} K}{2} \sigma_{11}^{2}(k)+\frac{d_{2} K}{2} \sigma_{11}^{2}(k)+\frac{d_{2} K}{2} \sigma_{12}^{2}(k)(N-K+K)^{2}+\frac{d_{2} K}{2} \sigma_{12}^{2}(k)(N-K+K)^{2},
\end{aligned}
$$

we have used the fact $(a+b)^{2} \leq 2\left(a^{2}+b^{2}\right)$ in second inequality. Further,

$$
\begin{aligned}
L V_{1} \leq & -\frac{b_{1}(k)(K+\xi(k))}{a_{1}(k)+\alpha(k) \xi(k)+K}+l(k)+\sigma_{21}^{2}(k)+d_{2} K \sigma_{11}^{2}(k)+2 d_{2} K^{3} \sigma_{12}^{2}(k)+F(N, k) \\
& +\frac{d_{1}(k) c_{1}(k) N P}{a_{1}(k)+\alpha(k) \xi(k)+N}+\frac{d_{2} K c_{1}(k) P}{a_{1}(k)+\alpha(k) \xi(k)+N}+\sigma_{22}^{2}(k) P^{2}-K \sum_{j \in \mathcal{M}} \gamma_{k j} d_{1}(j) \\
& +\sum_{j \neq k \in \mathcal{M}} \gamma_{k j}\left(\omega_{j}-\omega_{k}\right),
\end{aligned}
$$

where

$$
\begin{aligned}
F(N, k)= & -\frac{b_{1}(k)(N+\xi(k))}{a_{1}(k)+\alpha(k) \xi(k)+N}+\frac{b_{1}(k)(K+\xi(k))}{a_{1}(k)+\alpha(k) \xi(k)+K}+\frac{d_{1}(k) r(k)}{K} N(N-K)-\frac{d_{2} r(k)}{K}(N-K)^{2} \\
& +2 d_{2} K \sigma_{12}^{2}(k)(N-K)^{2}-\sum_{j \in \mathcal{M}} \gamma_{k j} d_{1}(j)(N-K) .
\end{aligned}
$$

Then the first and second derivatives of $F(N, k)$ with respect to $\mathrm{N}$ is

$$
\begin{aligned}
\frac{\partial F(N, k)}{\partial N}= & -\frac{b_{1}(k)\left(a_{1}(k)+\alpha(k) \xi(k)-\xi(k)\right)}{\left(a_{1}(k)+\alpha(k) \xi(k)+N\right)^{2}}+d_{1}(k) \frac{r(k)}{K}(2 N-K)-\frac{2 d_{2} r(k)}{K}(N-K) \\
& -\sum_{j \in \mathcal{M}} \gamma_{k j} d_{1}(j)+4 d_{2} K \sigma_{12}^{2}(k)(N-K)
\end{aligned}
$$


and

$$
\begin{aligned}
\frac{\partial^{2} F(N, k)}{\partial N^{2}} & =\frac{b_{1}(k)\left(a_{1}(k)+\alpha(k) \xi(k)-\xi(k)\right)}{\left(a_{1}(k)+\alpha(k) \xi(k)+N\right)^{3}}+2 d_{1}(k) \frac{r(k)}{K}-\frac{2 d_{2} r(k)}{K}+4 d_{2} K \sigma_{12}^{2}(k) \\
& \leq \frac{b_{1}(k)}{\left(a_{1}(k)+\alpha(k) \xi(k)\right)^{2}}+2 d_{1}(k) \frac{r(k)}{K}-\frac{2 d_{2} r(k)}{K}+4 d_{2} K \sigma_{12}^{2}(k) .
\end{aligned}
$$

By Lemma 4.1, $\left.\frac{\partial F(N, k)}{\partial N}\right|_{N=K}=0$. On the other hand, we have $\frac{\partial^{2} F(N, k)}{\partial N^{2}} \leq 0$ by the constant $d_{2}>$ $\max \left\{0, \max _{k \in \mathcal{M}}\left\{\frac{\frac{2 K b_{1}(k)}{K\left(a_{1}(k)+\alpha(k) \xi(k)\right)^{2}}+d_{1}(k) r(k)}{r(k)-2 K^{2} \sigma_{12}^{2}(k)}\right\}\right\}$ and the condition $\min _{k \in \mathcal{M}}\left\{r(k)-2 K^{2} \sigma_{12}^{2}(k)\right\}>0$ of Theorem 4.2 Thus $F(N, k) \leq F(K, k)=0$ and we obtain

$$
\begin{aligned}
L V_{1} \leq & -\frac{b_{1}(k)(K+\xi(k))}{a_{1}(k)+\alpha(k) \xi(k)+K}+l(k)+\sigma_{21}^{2}(k)+d_{2} K \sigma_{11}^{2}(k)+2 d_{2} K^{3} \sigma_{12}^{2}(k) \\
& +\frac{d_{1}(k) c_{1}(k) N P}{a_{1}(k)+\alpha(k) \xi(k)+N}+\frac{d_{2} K c_{1}(k) P}{a_{1}(k)+\alpha(k) \xi(k)+N}+\sigma_{22}^{2}(k) P^{2} \\
& -K \sum_{j \in \mathcal{M}} \gamma_{k j} d_{1}(j)+\sum_{j \neq k \in \mathcal{M}} \gamma_{k j}\left(\omega_{j}-\omega_{k}\right) \\
= & \lambda_{k}+\frac{d_{1}(k) c_{1}(k) N P}{a_{1}(k)+\alpha(k) \xi(k)+N}+\frac{d_{2} K c_{1}(k) P}{a_{1}(k)+\alpha(k) \xi(k)+N}+\sigma_{22}^{2}(k) P^{2} \\
& -K \sum_{j \in \mathcal{M}} \gamma_{k j} d_{1}(j)+\sum_{j \neq k \in \mathcal{M}} \gamma_{k j}\left(\omega_{j}-\omega_{k}\right) .
\end{aligned}
$$

Let $\mathbf{1}=(1, \ldots, 1)^{T} \in \mathbb{R}^{n}$ Now we define a vector $q=\left(q_{1}, q_{2}, \ldots, q_{n}\right)$ with $q_{k}=\lambda_{k}-K \sum_{j \in \mathcal{M}} \gamma_{k j} d_{1}(j)$, Since the generator matrix $\Gamma$ is irreducible, by Lemma 2.1 , for any $q$, there is $\omega=\left(\omega_{1}, \omega_{2}, \ldots, \omega_{n}\right)^{T}$ which is a solution of the system $\Gamma \omega=-q+\sum_{k=1}^{n} \pi_{k} q_{k} \mathbf{1}$. Then by the fact of (2.1) and $\pi \Gamma=0$

$$
\lambda_{k}-K \sum_{j \in \mathcal{M}} \gamma_{k j} d_{1}(j)+\sum_{j \neq k \in \mathcal{M}} \gamma_{k j}\left(\omega_{j}-\omega_{k}\right)=-\sum_{k \in \mathcal{M}} \pi_{k} \lambda_{k}=-\lambda .
$$

Substituting (4.4) into (4.3) leads to

$$
L V_{1} \leq-\lambda+\frac{\check{d}_{1} \check{c}_{1}}{\hat{a}_{1}+\hat{\alpha} \hat{\xi}} N P+\frac{d_{2} K \check{c}_{1}}{\hat{a}_{1}+\hat{\alpha} \hat{\xi}} P+\check{\sigma}_{22}^{2} P^{2}
$$

In addition, applying the generalized Itô's formula to $V_{2}$ yields

$$
\begin{aligned}
L V_{2} & =d_{3}\left(\frac{b_{1}(k)(N+\xi(k))}{a_{1}(k)+\alpha(k) \xi(k)+N}-l(k)\right) P^{v}+\frac{d_{3}(v-1)}{2}\left(\sigma_{21}(k)+\sigma_{22}(k) P\right)^{2} P^{v} \\
& \leq \frac{d_{3} b_{1}(k)}{a_{1}(k)+\alpha(k) \xi(k)} N P^{v}-\frac{d_{3}(1-v)}{2} \sigma_{22}^{2}(k) P^{v+2} \\
& \leq \frac{d_{3} \breve{b}_{1}}{\hat{a}_{1}+\hat{\alpha} \hat{\xi}} N P^{v}-\frac{d_{3}(1-v)}{2} \hat{\sigma}_{22}^{2} P^{v+2} .
\end{aligned}
$$

Moreover, by using the condition $\hat{l}>\frac{\breve{b}_{1} \breve{\xi}}{\hat{a}_{1}+\hat{\alpha} \hat{\xi}}$ in Theorem 4.2, we have

$$
\begin{aligned}
L V_{3} & =\frac{d_{2} K \check{c}_{1}}{\hat{\imath}\left(\hat{a}_{1}+\hat{\alpha} \hat{\xi}\right)-\breve{b}_{1} \check{\xi}}\left(\frac{b_{1}(k)(N+\xi(k))}{a_{1}(k)+\alpha(k) \xi(k)+N}-l(k)\right) P \\
& \leq \frac{d_{2} K \check{b}_{1} \check{c}_{1}}{\left(\hat{a}_{1}+\hat{\alpha} \hat{\xi}\right) \hat{l}\left(\hat{\imath}\left(\hat{a}_{1}+\hat{\alpha} \hat{\xi}\right)-\check{b}_{1} \check{\xi}\right)} N P-\frac{d_{2} K \check{c}_{1}}{\hat{a}_{1}+\hat{\alpha} \hat{\xi}} P .
\end{aligned}
$$


Similarly

$$
\begin{aligned}
L V_{4}= & N^{v}\left[r(k)\left(1-\frac{N}{K}\right)-\frac{c_{1}(k) P}{a_{1}(k)+\alpha(k) \xi(k)+N}\right]+\frac{v-1}{2}\left(\sigma_{11}(k)+\sigma_{12}(k) N\right)^{2} N^{v} \\
& +P^{v}\left[\frac{b_{1}(k)(N+\xi(k))}{a_{1}(k)+\alpha(k) \xi(k)+N}-l(k)\right]+\frac{v-1}{2}\left(\sigma_{21}(k)+\sigma_{22}(k) P\right)^{2} P^{v} \\
\leq & \check{r} N^{v}-\frac{1-v}{2} \hat{\sigma}_{12}^{2} N^{v+2}+\frac{\check{b}_{1}}{\hat{a}_{1}+\hat{\alpha} \hat{\xi}} N P^{v}-\frac{1-v}{2} \hat{\sigma}_{22}^{2} P^{v+2}
\end{aligned}
$$

Consequently, by (4.5)-(4.8), we can obtain

$$
\begin{aligned}
L V \leq & M\left[-\lambda+\frac{\check{d}_{1} \check{c}_{1}}{\hat{a}_{1}+\hat{\alpha} \hat{\xi}} N P+\check{\sigma}_{22}^{2} P^{2}+\frac{d_{3} \check{b}_{1}}{\hat{a}_{1}+\hat{\alpha} \hat{\xi}} N P^{v}-\frac{d_{3}(1-v)}{2} \hat{\sigma}_{22}^{2} P^{v+2}\right. \\
& \left.+\frac{d_{4} \check{b}_{1}}{\left(\hat{a}_{1}+\hat{\alpha} \hat{\xi}\right)} N P\right]+\check{r} N^{v}-\frac{1-v}{2} \hat{\sigma}_{12} N^{v+2}+\frac{\check{b}_{1}}{\hat{a}_{1}+\hat{\alpha} \hat{\xi}} N P^{v}-\frac{1-v}{2} \hat{\sigma}_{22} P^{v+2}
\end{aligned}
$$

It is easy to know that $\check{\sigma}_{22}^{2} P^{2}-\frac{d_{3}(1-v)}{2} \hat{\sigma}_{22}^{2} P^{v+2}$ gets the maximum at $P=\left(\frac{2}{v+2}\right)^{\frac{1}{v}}\left(\frac{2 \check{\sigma}_{22}^{2}}{d_{3}(1-v) \hat{\sigma}_{22}^{2}}\right)^{\frac{1}{v}}$, namely, $\check{\sigma}_{22}^{2} P^{2}-\frac{d_{3}(1-v)}{2} \hat{\sigma}_{22}^{2} P^{v+2} \leq\left(\frac{4 \check{\sigma}_{22}^{2}}{d_{3}(1-v)(v+2) \hat{\sigma}_{22}^{2}}\right)^{\frac{2}{v}} \frac{v}{v+2} \check{\sigma}_{22}^{2}$. Let $d_{3}=\frac{2^{\frac{v+4}{2}}\left(\frac{v}{v+2}\right)^{\frac{v}{2}} \check{\sigma}_{22}^{v+2}}{\lambda^{\frac{v}{2}}(v+2)(1-v) \hat{\sigma}_{22}^{2}}$, we have

$$
\begin{aligned}
L V \leq & M\left[-\frac{\lambda}{2}+\frac{\left(\check{d}_{1} \check{c}_{1}+d_{4} \check{b}_{1}\right)}{\hat{a}_{1}+\hat{\alpha} \hat{\xi}} N P+\frac{d_{3} \check{b}_{1}}{\hat{a}_{1}+\hat{\alpha} \hat{\xi}} N P^{v}\right] \\
& +\check{r} N^{v}-\frac{1-v}{2} \hat{\sigma}_{12} N^{v+2}+\frac{\check{b}_{1}}{\hat{a}_{1}+\hat{\alpha} \hat{\xi}} N P^{v}-\frac{1-v}{2} \hat{\sigma}_{22} P^{v+2}
\end{aligned}
$$

Define a bounded closed set $D_{\varepsilon}=\left\{(N, P) \in \mathbb{R}_{+}^{2}: \varepsilon<N<\frac{1}{\varepsilon}, \varepsilon<P<\frac{1}{\varepsilon}\right\}$, where $\varepsilon$ is a sufficiently small number. In the set $\mathbb{R}_{+}^{2} \backslash D_{\varepsilon}$, we can choose sufficiently small $\varepsilon$ such that the following conditions hold

$$
\begin{gathered}
0<\varepsilon<F . \\
0<\varepsilon<G_{1} . \\
0<\varepsilon<F^{\frac{1}{v}} . \\
0<\varepsilon<G_{2}^{\frac{1}{v}} . \\
-\frac{M \lambda}{2}-\frac{1-v}{4} \hat{\sigma}_{12} \frac{2^{v+2}}{\varepsilon}+I_{1} . \\
-\frac{M \lambda}{2}-\frac{1-v}{4} \hat{\sigma}_{22} \frac{1}{\varepsilon^{v+2}}+I_{2} .
\end{gathered}
$$

where $F=-\frac{M \lambda\left(\hat{a}_{1}+\hat{\alpha} \hat{\xi}\right)}{4 M\left(\check{d}_{1} \check{c}_{1}+d_{4} \ddot{b}_{1}+d_{3} \check{b}_{1}\right)+\check{b}_{1}}, G_{1}=\left(\frac{(1-v) \hat{\sigma}_{12}\left(\hat{a}_{1}+\hat{\alpha} \hat{\xi}\right)}{4 M\left(\check{d}_{1} \check{c}_{1}+d_{4} \breve{b}_{1}+d_{3} \breve{b}_{1}\right)+\check{b}_{1}}\right)^{\frac{1}{v}}, G_{2}=\left(\frac{(1-v) \hat{\sigma}_{22}\left(\hat{a}_{1}+\hat{\alpha} \hat{\xi}\right)}{4 M\left(\check{d}_{1} \check{c}_{1}+d_{4} \ddot{b}_{1}+d_{3} \breve{b}_{1}\right)+\breve{b}_{1}}\right)^{\frac{1}{v}}, I_{1}$ and $I_{2}$ are positive constants which will be given explicitly in the following. For convenience, we can divide $\mathbb{R}_{+}^{2} \backslash D_{\varepsilon}$ 
into four domains,

$$
\begin{array}{ll}
D_{\varepsilon}^{1}=\left\{(N, P) \in \mathbb{R}_{+}^{2}: 0<N<\varepsilon\right\}, & D_{\varepsilon}^{2}=\left\{(N, P) \in \mathbb{R}_{+}^{2}: 0<P<\varepsilon\right\}, \\
D_{\varepsilon}^{3}=\left\{(N, P) \in \mathbb{R}_{+}^{2}: N<\frac{1}{\varepsilon}\right\}, & D_{\varepsilon}^{4}=\left\{(N, P) \in \mathbb{R}_{+}^{2}: P<\frac{1}{\varepsilon}\right\} .
\end{array}
$$

Clearly, $D_{\varepsilon}^{c}=D_{\varepsilon}^{1} \cup D_{\varepsilon}^{2} \cup D_{\varepsilon}^{3} \cup D_{\varepsilon}^{4}$. Next, we will prove that $L V(N, P, k) \leq-1$ for any $(N, P, k) \in D_{\varepsilon}^{c} \times \mathcal{M}$, which is equivalent to proving it on the above four domains, respectively.

Case 1. For any $(N, P, k) \in D_{\varepsilon}^{1} \times \mathcal{M}$, since $N P^{v} \leq \varepsilon\left(1+P^{v+2}\right)$ and $N P \leq \varepsilon\left(1+P^{v+2}\right)$, we obtain

$$
\begin{aligned}
L V \leq & -\frac{M \lambda}{8}+\left(-\frac{M \lambda}{4}+\frac{\hat{a}_{1}+\hat{\alpha} \hat{\xi}}{4 M\left(\check{d}_{1} \check{c}_{1}+d_{4} \dot{b}_{1}+d_{3} \check{b}_{1}\right)+\check{b}_{1}} \varepsilon\right)-\frac{1-v}{4} \hat{\sigma}_{12} N^{v+2} \\
& +\left(\frac{\hat{a}_{1}+\hat{\alpha} \hat{\xi}}{4 M\left(\check{d}_{1} \check{c}_{1}+d_{4} \dot{b}_{1}+d_{3} \breve{b}_{1}\right)+\check{b}_{1}} \varepsilon-\frac{1-v}{4} \hat{\sigma}_{22}\right) P^{v+2} \\
& +\left[-\frac{M \lambda}{8}+\sup _{(N, P) \in \mathbb{R}_{+}^{2}}\left\{-\frac{1-v}{4}\left(\hat{\sigma}_{12} N^{v+2}+\hat{\sigma}_{22} P^{v+2}\right)+\check{r} N^{v}\right\}\right] .
\end{aligned}
$$

Since $M=\frac{4}{\lambda} \max \left\{2, \sup _{(N, P) \in \mathbb{R}_{+}^{2}}\left\{-\frac{1-v}{4}\left(\hat{\sigma}_{12} N^{v+2}+\hat{\sigma}_{22} P^{v+2}\right)+\check{r} N^{v}\right\}\right\}$, we have $\frac{M \lambda}{8} \geq 1$. Thus

$$
L V \leq-\frac{M \lambda}{8}-\frac{1-v}{4} \hat{\sigma}_{12} N^{v+2} \leq-1
$$

which follows from the definition of $\mathrm{M},(4.10)$ and (4.11).

Case 2. For any $(N, P, k) \in D_{\varepsilon}^{2} \times \mathcal{M}$, since $N P^{v} \leq \varepsilon^{v}\left(1+N^{v+2}\right)$ and $N P \leq \varepsilon\left(1+N^{v+2}\right) \leq \varepsilon^{v}\left(1+N^{v+2}\right)$, we obtain

$$
\begin{aligned}
L V \leq & -\frac{M \lambda}{8}+\left(-\frac{M \lambda}{4}+\frac{\hat{a}_{1}+\hat{\alpha} \hat{\xi}}{4 M\left(\check{d}_{1} \check{c}_{1}+d_{4} \check{b}_{1}+d_{3} \check{b}_{1}\right)+\check{b}_{1}} \varepsilon^{v}\right)-\frac{1-v}{4} \hat{\sigma}_{22} P^{v+2} \\
& +\left(\frac{\hat{a}_{1}+\hat{\alpha} \hat{\xi}}{4 M\left(\check{d}_{1} \check{c}_{1}+d_{4} \breve{b}_{1}+d_{3} \check{b}_{1}\right)+\check{b}_{1}} \varepsilon^{v}-\frac{1-v}{4} \hat{\sigma}_{12}\right) N^{v+2} \\
& +\left[-\frac{M \lambda}{8}+\sup _{(N, P) \in \mathbb{R}_{+}^{2}}\left\{-\frac{1-v}{4}\left(\hat{\sigma}_{12} N^{v+2}+\hat{\sigma}_{22} P^{v+2}\right)+\check{r} N^{v}\right\}\right] \\
\leq & -\frac{M \lambda}{8}-\frac{1-v}{4} \hat{\sigma}_{12} P^{v+2} \leq-1,
\end{aligned}
$$

which follows from the definition of M, (4.12) and (4.13).

Case 3. For any $(N, P, k) \in D_{\varepsilon}^{3} \times \mathcal{M}$, we have

$$
\begin{aligned}
L V \leq & -\frac{M \lambda}{2}-\frac{1-v}{4} \hat{\sigma}_{12} N^{v+2}+\frac{M\left(\check{d}_{1} \check{c}_{1}+d_{4} \check{b}_{1}\right)}{\hat{a}_{1}+\hat{\alpha} \hat{\xi}} N P+\frac{\left(d_{3} M+1\right) \check{b}_{1}}{\hat{a}_{1}+\hat{\alpha} \hat{\xi}} N P^{v} \\
& +\check{r} N^{v}-\frac{1-v}{4} \hat{\sigma}_{12} N^{v+2}-\frac{1-v}{2} \hat{\sigma}_{22} P^{v+2} \\
\leq & -\frac{M \lambda}{2}-\frac{1-v}{4} \hat{\sigma}_{12} N^{v+2}+I_{1} \leq-\frac{M \lambda}{2}-\frac{1-v}{4} \hat{\sigma}_{12} \frac{1}{\varepsilon^{v+2}}+I_{1} \\
\leq & -1 .
\end{aligned}
$$



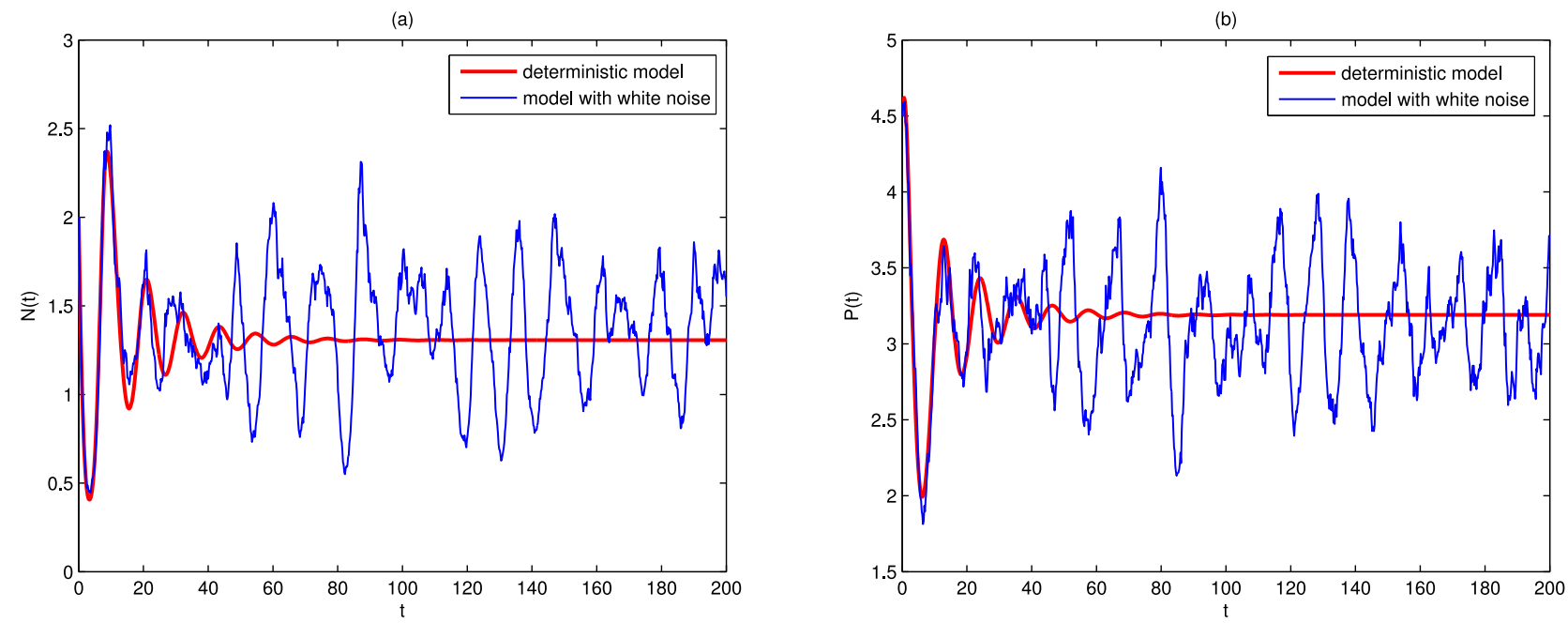

Figure 1 . The simple paths of the process $(N(t), P(t))$. The red lines represent populations in deterministic model and the blue lines represent populations in stochastic model with white noise,respectively.

which follows from (4.13).

$$
\begin{aligned}
I_{1}= & \sup _{(N, P) \in \mathbb{R}_{+}^{2}}\left\{-\frac{1-v}{4} \hat{\sigma}_{12} N^{v+2}-\frac{1-v}{2} \hat{\sigma}_{22} P^{v+2}+\frac{M\left(\check{d}_{1} \check{c}_{1}+d_{4} \check{b}_{1}\right)}{\hat{a}_{1}+\hat{\alpha} \hat{\xi}} N P\right. \\
& \left.+\frac{\left(d_{3} M+1\right) \check{b}_{1}}{\hat{a}_{1}+\hat{\alpha} \hat{\xi}} N P^{v}+\check{r} N^{v}\right\} .
\end{aligned}
$$

Case 4. For any $(N, P, k) \in D_{\varepsilon}^{4} \times \mathcal{M}$, we have

$$
\begin{aligned}
L V \leq & -\frac{M \lambda}{2}-\frac{1-v}{4} \hat{\sigma}_{12} P^{v+2}+\frac{M\left(\check{d}_{1} \check{c}_{1}+d_{4} \check{b}_{1}\right)}{\hat{a}_{1}+\hat{\alpha} \hat{\xi}} N P+\frac{\left(d_{3} M+1\right) \check{b}_{1}}{\hat{a}_{1}+\hat{\alpha} \tilde{\xi}} N P^{v} \\
& +\check{r} N^{v}-\frac{1-v}{2} \hat{\sigma}_{12} N^{v+2}-\frac{1-v}{4} \hat{\sigma}_{22} P^{v+2} \\
\leq & -\frac{M \lambda}{2}-\frac{1-v}{4} \hat{\sigma}_{22} P^{v+2}+I_{2} \leq-\frac{M \lambda}{2}-\frac{1-v}{4} \hat{\sigma}_{22} \frac{1}{\varepsilon^{v+2}}+I_{2} \\
\leq & -1 .
\end{aligned}
$$

which follows from (4.14).

$$
\begin{aligned}
I_{2}= & \sup _{(N, P) \in \mathbb{R}_{+}^{2}}\left\{-\frac{1-v}{2} \hat{\sigma}_{12} N^{v+2}-\frac{1-v}{4} \hat{\sigma}_{22} P^{v+2}+\frac{M\left(\check{d}_{1} \check{c}_{1}+d_{4} \check{b}_{1}\right)}{\hat{a}_{1}+\hat{\alpha} \hat{\xi}} N P\right. \\
& \left.+\frac{\left(d_{3} M+1\right) \check{b}_{1}}{\hat{a}_{1}+\hat{\alpha} \hat{\xi}} N P^{v}+\check{r} N^{v}\right\} .
\end{aligned}
$$

Obviously, from (4.16)-(4.19), we can see that for a sufficiently small $\varepsilon, L V \leq-1$ for all $(P, N, \Lambda) \in \mathbb{R}_{+}^{2} \backslash D_{\varepsilon}$. Hence (c) in Lemma 2.3 is satisfied. According to Lemma 2.3, we can conclude that system (1.2) is ergodic and admits a unique stationary distribution. This completes the proof. 


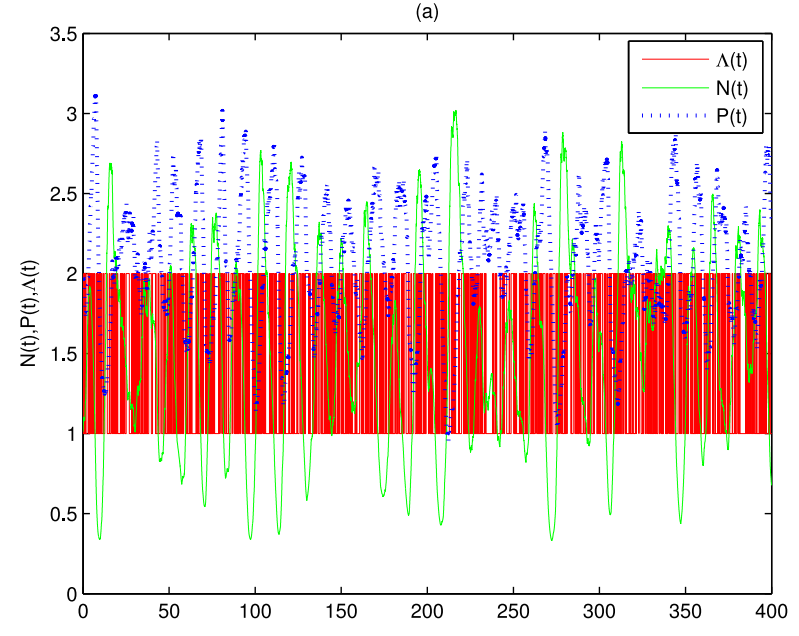

(b) density of $\mathrm{N}$

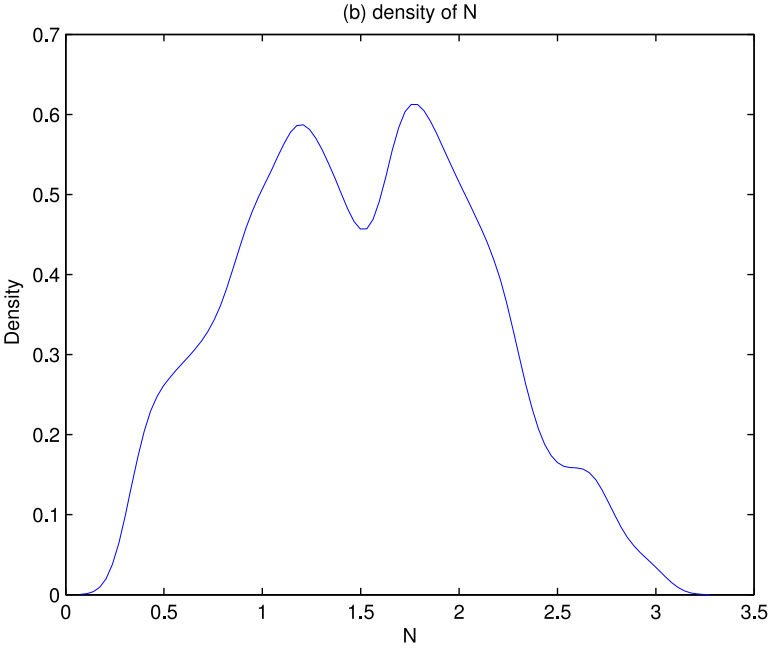

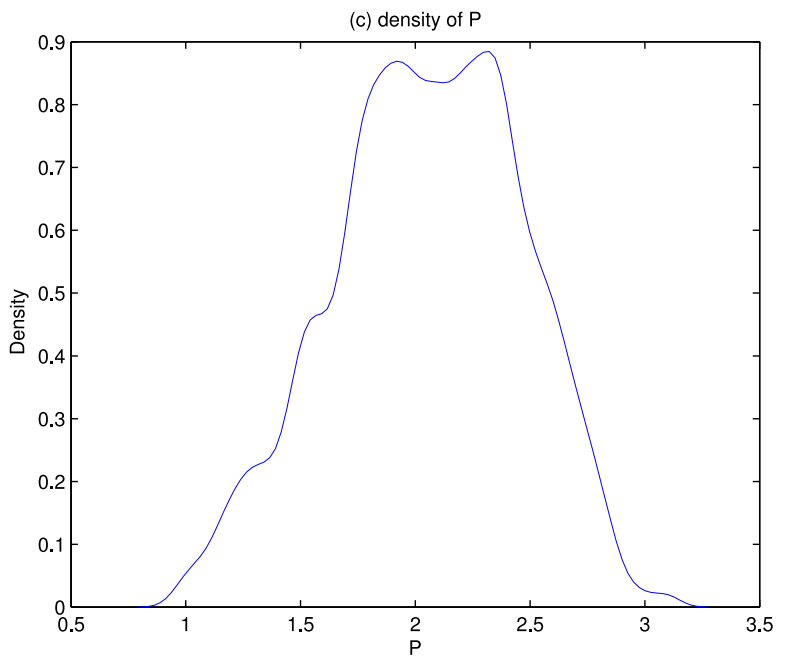

FIGURE 2. The system (1.2) has ergodic property. The subgraphs (a) depicts the simple path of $(N(t), P(t))$ for the stochastic system (1.2) and the Markovian chain, where the noises are small enough;

(b) and (c) represent the unique density functions of populations $N(t)$ and $P(t)$, respectively.

\section{Numerical SimULATiONS}

In this section, we illustrate the influence of white noise and Markovian switching. Using Milstein's Higher Order Method in [14], we get the discretization transformation of system (1.2) as

$$
\left\{\begin{aligned}
x_{j+1}= & x_{j}+x_{j}\left[r(k)\left(1-\frac{x_{j}}{K}\right)-\frac{\left.c_{1}(k)\right) y_{j}}{a_{1}(k)+\alpha(k) \xi(k)+x_{j}}\right] \Delta t \\
& +x_{j}\left(\sigma_{11}(k)+\sigma_{12}(k) x_{j}(t)\right) \sqrt{\triangle t}+\frac{x_{j}^{2}\left(\sigma_{11}(k)+\sigma_{12}(k) x_{j}\right)^{2}}{2}\left(\varepsilon_{1 j}^{2} \Delta t-\triangle t\right), \\
y_{j}+1= & y_{j}\left[\frac{b_{1}(k)\left(x_{j}+\xi(k)\right)}{a_{1}(k)+\alpha(k) \xi(k)+x_{j}}-l(k)\right] \triangle t \\
& +y_{j}(t)\left(\sigma_{21}(k)+\sigma_{22}(k) y_{j}\right) \sqrt{\triangle t}+\frac{y_{j}^{2}\left(\sigma_{21}(k)+\sigma_{22}(k) y_{j}(t)\right)^{2}}{2}\left(\varepsilon_{2 j}^{2} \triangle t-\triangle t\right),
\end{aligned}\right.
$$



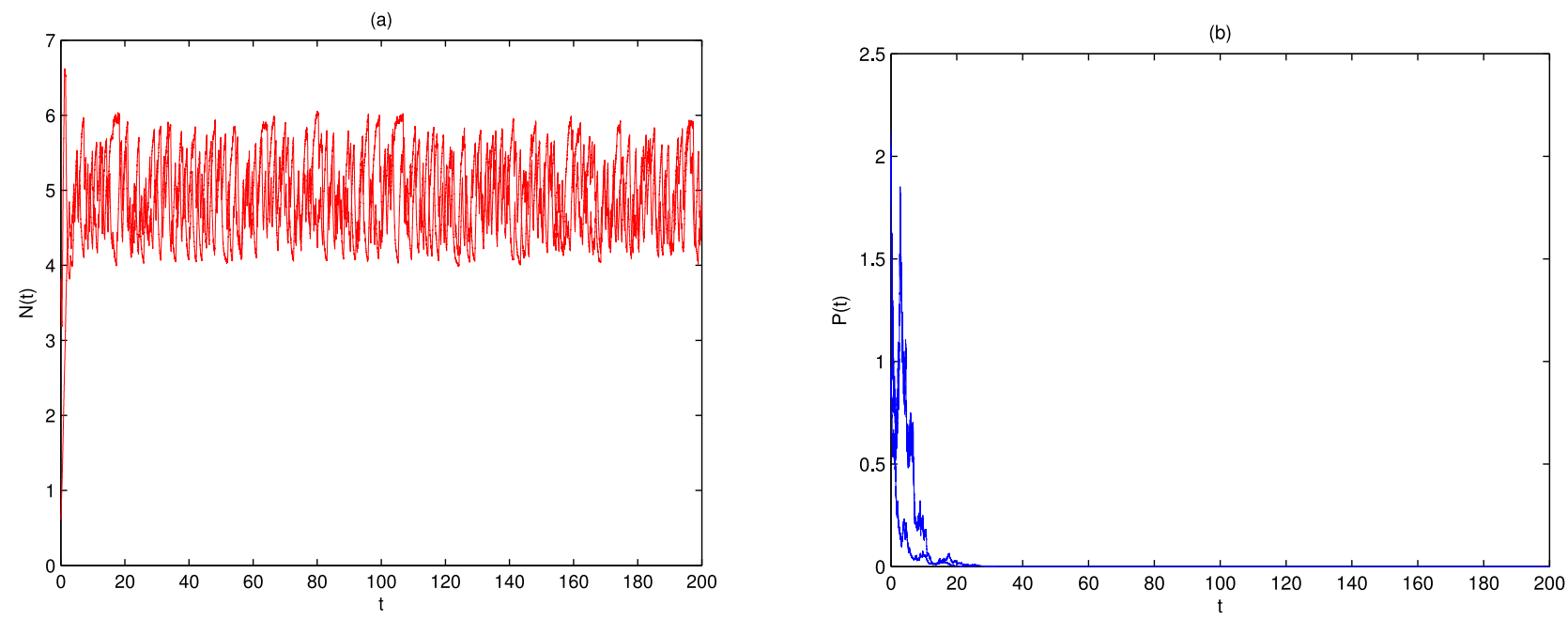

Figure 3. Only the predator population $P(t)$ will go extinct. (a) and (b) are the simple paths of the process $(N(t), P(t))$ corresponding to the stochastic system (1.2), respectively.
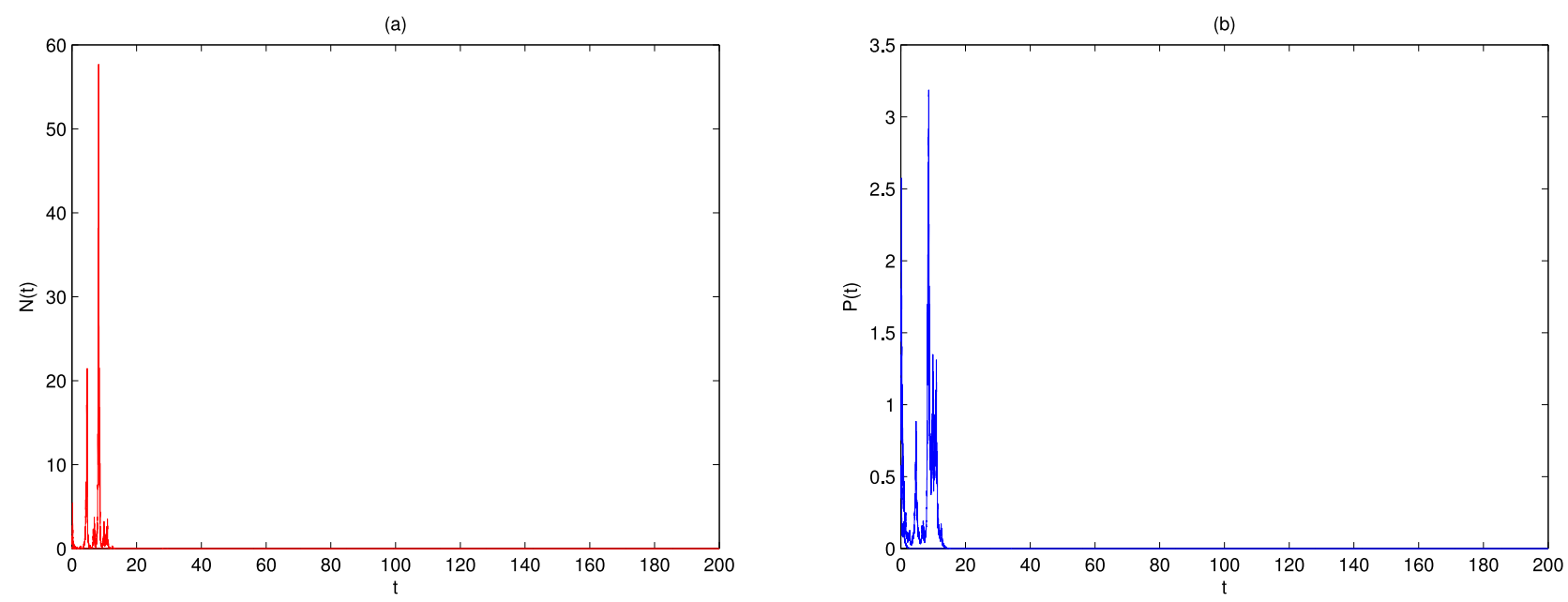

FigurE 4. Both the prey and predator populations will go extinct. (a) and (b) are the simple paths of the process $(N(t), P(t))$ corresponding to the stochastic system (1.2), respectively.

where the time increment $\Delta t$ and $\varepsilon_{1 j}, \varepsilon_{2 j}$ are independent Gaussian random variables with distribution For convenience, we assume the Markovian chain $\Lambda(t)$ takes values in $\mathcal{M}=\{1,2\}$, the generator $\Gamma=\left[\begin{array}{cc}-3 & 3 \\ 2 & -2\end{array}\right]$, so $\pi=(0.4,0.6)$. In system $(1.2)$, we choose appropriate parameters: $(r(1), r(2))=$ $(2,1.5) ; \quad\left(c_{1}(1), c_{1}(2)\right)=(1.4,1.6), \quad\left(a_{1}(1), a_{1}(2)\right)=(1,1.5), \quad\left(b_{1}(1), b_{1}(2)\right)=(1,1.3), \quad(\alpha(1), \alpha(2))=$ $(0.5,0.4),(l(1), l(2))=(0.4,0.5)(\xi(1), \xi(2))=(0.02,0.025), K=4[8]$.

1. At the outset of our numerical simulation, we deal with the case only with white noise. Hence system (1.2) is reduced to the model in [8]. Let $r=2 ; c_{1}=1.2, a_{1}=1, b_{1}=1.3, \alpha=0.5, l=0.4, \xi=0.025, K=4$. $\sigma_{11}=0.006, \sigma_{12}=0.001, \sigma_{21}=0.005, \sigma_{22}=0.001$. Initial values are taken at $(2,3)$. By making use of the conditions of Theorem 5.4 in [8], we obtain that the prey population and the predator population coexist for a long time (see Fig. 1). So we conclude that low intensity white noise does not cause extinction and keeps 

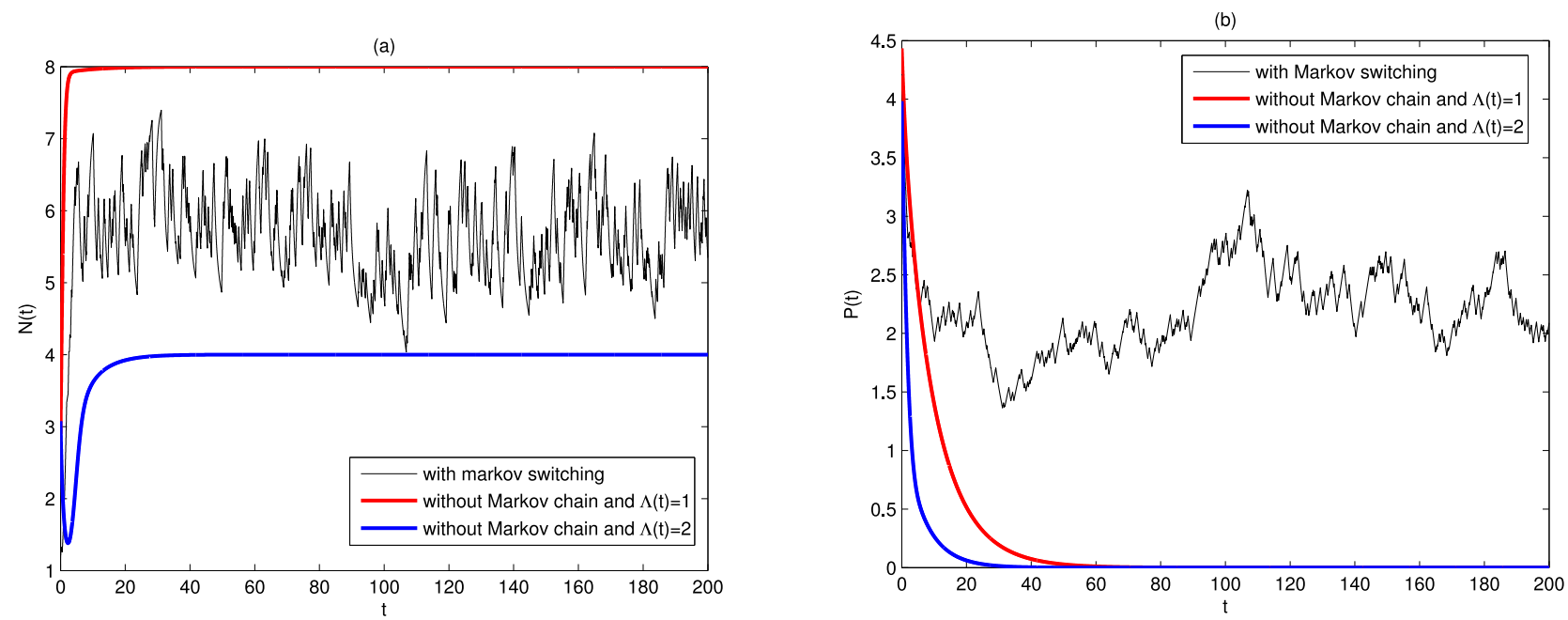

Figure 5. The black lines represent the paths of $N(t)$ and $P(t)$ switching according to the movement of $\Lambda(t)$. The red lines represent the paths of the process $(N(t), P(t))$ only in sate 1 ; the blue lines represent the paths of the process $(N(t), P(t))$ only in state 2 .

(a)

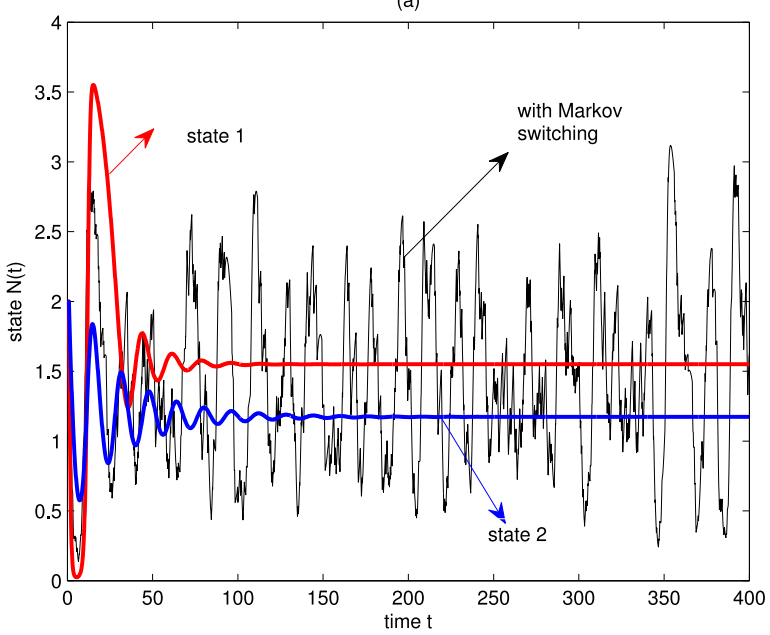

(b)

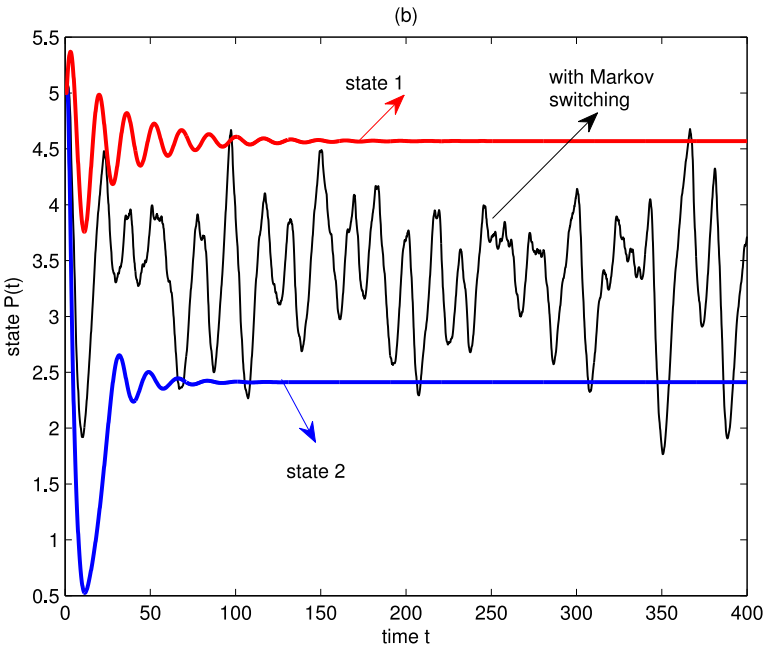

FigURE 6 . The black lines represent the paths of $N(t)$ and $P(t)$ switching according to the movement of $\Lambda(t)$. The red lines represent the paths of the process $(N(t), P(t))$ only in sate 1 ; the blue lines represent the paths of the process $(N(t), P(t))$ only in state 2 .

stochastic process $(N(t), P(t))$ moving up and down randomly in a small neighborhood of the corresponding deterministic system.

2 . Now we consider the case with Markovian switching. Let $\left(\sigma_{11}(1), \sigma_{11}(2)\right)=(0.006,0.005),\left(\sigma_{12}(1), \sigma_{12}(2)\right)=$ $(0.001,0.002),\left(\sigma_{21}(1), \sigma_{21}(2)\right)=(0.005,0.004),\left(\sigma_{22}(1), \sigma_{22}(2)\right)=(0.001,0.001)$ and $d_{2}=10^{3}$ which satisfy the conditions of Theorem 4.2: $\hat{l}=0.5>\frac{\breve{b}_{1} \tilde{\xi}}{\hat{a}_{1}+\hat{\alpha} \xi}=0.0167, \max _{k \in \mathcal{M}}\left\{r(k)-2 K^{2} \sigma_{12}^{2}(k)\right\}=1.4999>0$ and $d_{2}>$ $\max _{k \in \mathcal{M}}\left\{\frac{\frac{K b_{1}(k)}{2\left(a_{1}(k)+\alpha(k) \xi(k)\right)^{2}}+d_{1}(k) r(k)}{r(k)-2 K^{2} \sigma_{12}^{2}(k)}\right\}=0.9017$ such that the following condition holds $\lambda=\sum_{k \in \mathcal{M}} \pi_{k} \lambda_{k}=0.0899>0$.

One can see that there exists a unique stationary distribution of system (1.2) (see Fig. 2). 
3. If $\left(\sigma_{11}(1), \sigma_{11}(2)\right)=(0.006,0.005),\left(\sigma_{12}(1), \sigma_{12}(2)\right)=(0.001,0.002),\left(\sigma_{22}(1), \sigma_{22}(2)\right)=(0.001,0.001)$, we decrease the parameters $\left(\sigma_{21}(1), \sigma_{21}(2)\right)=(1,1.5)$ satisfying the condition of (ii): $\sum_{i=1}^{2} \pi_{i}\left(l(i)+\frac{\sigma_{21}^{2}(i)}{2}-\frac{b_{1}(i) \xi(i)}{a_{1}(i)+\alpha(i) \xi(i)}\right)=-1.0914<0$ in Theorem 3.2, we can see that the predator population will become extinct about $t=28$ (see Fig. 3). Based on this, now we continue to decrease the parameters $\left(\sigma_{11}(1), \sigma_{11}(2)\right)=(2,2.1)$, so that the conditions of (i) in Theorem $4.2: \sum_{i=1}^{2} \pi_{i}\left(r(i)-\frac{\sigma_{11}^{2}(i)}{2}\right)=-0.3525<0$ and $\sum_{i=1}^{2} \pi_{i}\left(l(i)+\frac{\sigma_{21}^{2}(i)}{2}-\frac{b_{1}(i) \xi(i)}{a_{1}(i)+\alpha(i) \xi(i)}\right)=-0.1878<0$ satisfy. So both prey and predator populations will go extinct (see Fig. 4). Besides,we can see that the prey population will go extinct about $t=10$ and the predator population will go extinct about $t=16$.

4. If $\sigma_{i j}=0 \quad(i, j=1,2),\left(c_{1}(1), c_{1}(2)\right)=(0.9,0.8),\left(b_{1}(1), b_{1}(2)\right)=(0.5,0.36) \quad(l(1), l(2))=(0.5,0.4)$, $(\xi(1), \xi(2))=(0.12,0.02)$, the other parameters remain the same. By computation, we can obtain that the predator will extinct if there is only one state 1 or 2 while the predator is persistent if stochastic process $(N(t), P(t))$ switches back and forth from one state 1 to another state 2 according to the movement of $\Lambda(t)$ (see Fig. 5).

5. If $\sigma_{i j}=0(i, j=1,2),\left(c_{1}(1), c_{1}(2)\right)=(1.7,1.4),\left(b_{1}(1), b_{1}(2)\right)=(0.9,0.7)$ the other parameters remain the same. By computation, we can obtain that the predator will be persistent all the way (see Fig. 6).

\section{Conclusion}

In this paper, we have considered a stochastic predator-prey model with additional food for predator. All parameters but carrying capacity have been perturbed with telephone noise. We have also introduced nonlinear white noise on the birth rate of prey and on the death rate of predator. Considering the environmental noise we first give the existence and uniqueness theorem of global positive solution of the system. Since the proof is standard, we omit it. Then the conditions for extinction of prey and predator have been obtained (Thm. 3.2). It is proved that the system (1.2) has a unique stationary distribution when the noise intensity is significantly small (Thm. 4.2). Our investigation shows that the white noise, Markovian chain and additional food may cause great influence on the survival of species as follows:

(1) The white noise is harmful to the survival of species. It is obtained that small white noise keeps stochastic process $(N(t), P(t))$ moving up and down randomly in a small neighborhood of the corresponding deterministic system (see Fig. 1). When white noise intensity is smaller, system (1.2) has a unique stationary distribution (see Fig. 2a-c). But If we increase the white noise $\sigma_{22}$, the predator become extinct at time $t=28$ (see Fig. 3b); we further increase the white noise $\sigma_{11}$, the prey becomes extinct at $t=10$, then the predator also becomes extinct at time $t=16$ (see Fig. 4). So larger white noise intensity can cause the extinction of the population and the extinction of prey population speeds up predator population to extinction.

(2) The Markovian chain $\boldsymbol{\Lambda}(\mathbf{t})$ can suppress the extinction of species. Figure 2a depicts the movement of the Markovian chain $\Lambda(t)$ in $\mathcal{M}=\{1,2\}$. Figure $2 \mathrm{~b}$ and $\mathrm{c}$ display the density functions of $N$ and $P$. Figures 5 and 6 describe that the stochastic process switches back and forth from one state 1 to another state 2 according to the movement of $\Lambda(t)$ and only stays in state 1 or 2 . The difference is that Figure 6 is also affected by white noise while Figure 5 is not. Clearly, in Figure $5 \mathrm{a}$, the black line is located between the red line and the blue line. In Figure 5b, the black line oscillates between 1.5 and 3, the red line and the blue line go to zero, that is to say, the predator population with switching is persistence but goes extinct in only one state 1 or 2 . In Figure $6 \mathrm{~b}$, the black line oscillates between the red line and the blue line, that is to say, the switching state is located between state 1 and state 2 . Therefore, the existence of colored noise reduces the risk of species extinction.

Finally, we like to mention that we deal with state-independent Markovian switching model in our paper, in fact, for any $i, j \in \mathcal{M}, x \in \mathbb{R}_{+}^{2}$,

$$
P\left\{\Lambda(t+\delta)=j \mid \Lambda(t)=i, X_{t}=x\right\}=\left\{\begin{array}{cc}
\gamma_{i j}(x) \delta+o(\delta), & \text { if } \quad i \neq j \\
1+\gamma_{i j}(x) \delta+o(\delta), & \text { if } \quad i=j
\end{array}\right.
$$


whenever $\delta \rightarrow 0$, where $X_{t}=\left(N_{t}, P_{t}\right) \in \mathbb{R}_{+}^{2}$. But there are essential challenges to cope with the model under state-independent Markovian switching. One of the challenges is that the classical ergodic theorem for continuous-time Markovian chains does not work any more due to the fact that $\Lambda(t)$ is merely a jump process rather than a Markovian process. we will get over such difficulty in near future.

Acknowledgements. The authors would like to thank the anonymous reviewers for their valuable comments and suggestions which have improved the quality of this paper. This work was supported by the Graduate Joint Training Program of the Guangdong Educational Department, China and the Innovation Research for the postgraduates of Guangzhou University under Grant No. 2018GDJC-D02.

\section{REFERENCES}

[1] P. Aguirre, E. González-Olivares, S. Torres, Stochastic predator-prey model with Allee effect on prey. Nonlinear Anal. Real World Appl. 14 (2013) 768-779.

[2] W. Allee, Animal aggregations: A study in general sociology, University of Chicago Press, Chicago (1931).

[3] J. Bao, J. Shao, Permanence and extinction of regime-switching predator-prey models. J. Math. Anal. 48 (2015) $725-739$.

[4] Y. Cai, X. Mao, A stochastic prey-predator model with time-dependent delays. Appl. Math. Model. 64 (2018) $357-371$.

[5] J. Chattopadhyay, O. Arino, A predator-prey model with disease in the prey. Nonlinear Anal. 36 (1999) $747-766$.

[6] T. Chowdhury, S. Chakraborty, J. Chattopadhyay, Migratory effect of middle predator in a tri-trophic food chain model. Math. Methods Appl. Sci. 33 (2010) 1699-1711.

[7] N. Dang, N. Du, T. Ton, Asymptotic behavior of predator-prey systems perturbed by white noise. Acta Appl. Math. 115 (2011) 351-370.

[8] A. Das, G. Samanta, Stochastic prey-predator model with additional food for predator. Physica A 512 (2018) $121-141$.

[9] P. DeBach, Biological Control by Natural Enemies, Cambridge University Press, UK, (1974).

[10] S. Ghorai, S. Poria, Impacts of additional food on diffusion induced instabilities in a predator-prey system with mutually interfering predator. Chaos Solitons Fractals 103 (2017) 68-78.

[11] E. González-Olivares, R. Ramos-Jiliberto, Dynamic consequences of prey refuges in a simple model system: more prey, fewer predators and enhanced stability. Ecol. Model. 166 (2003) 135-146.

[12] Q. Han, D. Jiang, C.Ji, Analysis of a delayed stochastic predator-prey model in a polluted environment. Appl. Math. Model. 38 (2014) 3067-3080.

[13] R.Z. Hasminskii, Stochastic Stability of Differential Equations. Sijthoff and Noordhoff, Maryland (1980).

[14] D. Higham, An algorithmic introduction to numerical simulation of stochastic differential equations. SIAM. Rev. 43 (2001) $525-546$.

[15] C. Holling, Some characteristics of simple types of predation and parasitism. Mem. Entomol. Soc. Canada 46 (1965) 1-60.

[16] D. Kumar, S. Chakrabarty, A predator-prey model with additional food supply to predators: dynamics and applications. $J$. Comp. Appl. Math. 37 (2018) 763-784.

[17] A. Lahrouz, A. Settati, P.S. Mandal, Dynamics of a switching diffusion modified Leslie Gower Predator-prey system with Beddington-DeAngelis functional response. Nonlinear Dynam. 85 (2016) 853-870.

[18] M. Liu, X. He, J. Yu, Dynamics of a stochastic regime-switching predator-prey model with harvesting and distributed delays. Nonlinear Anal. Hybrid. 28 (2018) 87-104.

[19] R. Liu, G. Liu, Analysis of a stochastic predator-prey population model with Allee effect and jumps. J. Inequal. Appl. 60 (2019) 1-16.

[20] A. Lotka, Analytical note on certain rhythmic relations in organic systems. Proc. Natl. Acad. Sci. 6 (1920) $410-415$.

[21] X. Mao, Stochastic Differential Equations and Applications. Horwood Publishing, Chichester (1997).

[22] R. May, Stability in randomly fluctuating versus deterministic environments. Am. Nat. 107 (1973) 621-650.

[23] J. Margaritopoulos, J. Tsitsipis, D. Perdikis, Biological characteristics of the mirids Macrolophus costalis and Macroplophus pygmaeus preying on the tobacco form of Myzus persicae (Hemiptera: Aphididae). B. Entomol. Res. 93 (2003) 39.

[24] B. Sahoo, S. Poria, Effects of additional food in a delayed predator-prey model. Math. Biosc. 261 (2015) 62-73.

[25] P. Srinivasu, B. Prasad, Role of quantity of additional food to predators as a control in predator-prey systems with relevance to pest management and biological conservation. Bull. Math. Biol. 73 (2011) 2249-2276.

[26] P. Srinivasu, D. Vamsi, V. Ananth, Additional food supplements as a tool for biological conservation of predator-prey systems involving type III functional response: A qualitative and quantitative investigation. J. Theor. Biol. 455 (2018) 303-318.

[27] V. Volterra, Variazioni e fluttuazioni del numero d'individui in specie d'animali conviventi. Mem. Acad. Lincei 2 (1989) 31-113.

[28] G. Yin, C. Zhu, Asymptotic properties of hybrid diffusion systems. SIAM J. Control Optim. 46 (2007) 1155-1179.

[29] G. Yin, C. Zhu, Hybrid Switching Diffusions Properties and Applications. Springer-Verlag, New York (2009).

[30] L. Zu, D. Jiang, D. O'Regand, T. Hayat, Ergodic property of a Lotka-Volterra predator-prey model with white noise higher order perturbation under regime switching. Appl. Math. Comput. 330 (2018) 93-102. 